\title{
Chiral Brønsted Acid-catalyzed Enantioselective Mannich-type Reaction
}

\author{
Masahiro Yamanaka, ${ }^{*} \ddagger$ Junji Itoh, $\dagger$ \\ Kohei Fuchibe, $\uparrow$ and Takahiko Akiyama, ${ }^{*} \dagger$ \\ Department of Chemistry, Faculty of Science, Gakushuin University, \\ 1-5-1 Mejiro, Toshima-ku, Tokyo 171-8588, Japan, $\dagger$ \\ Department of Chemistry, Faculty of Science, Rikkyo University, \\ 3-34-1 Nishi-Ikebukuro, Toshima-ku Tokyo 171-8501, Japan.‡ \\ takahiko.akiyama@gakushuin.ac.jp \\ myamanaka@rikkyo.ac.jp
}

\section{Supporting Information}

\section{Contents}

Additional Results$-\mathrm{S} 2$

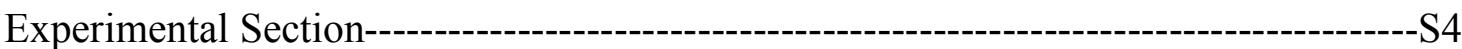

Cartesian Coordinates and Absolute Electronic Energies of Statinary Points-----------S24 

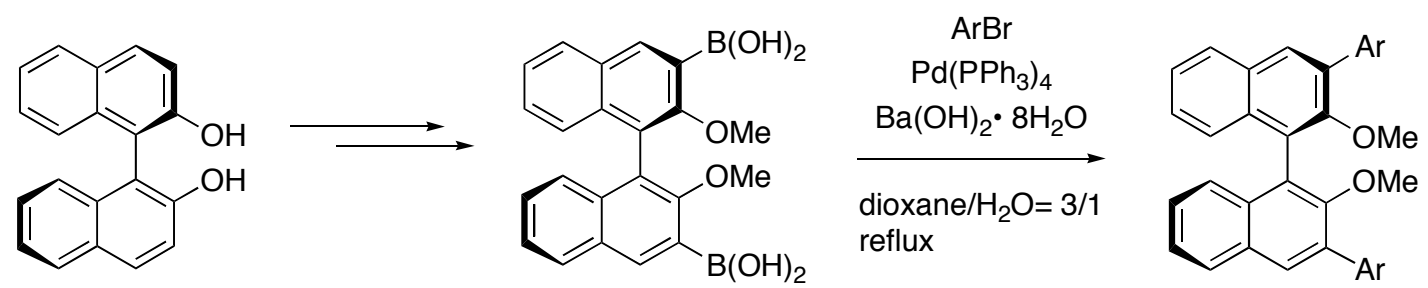

$(70 \%)$

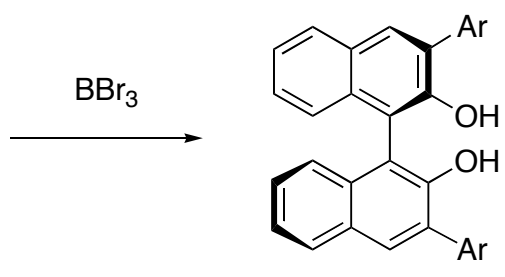

$(70 \%)$
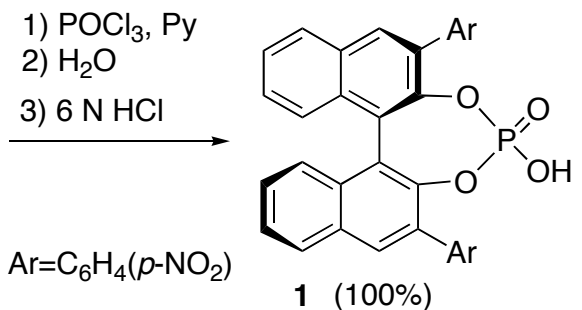

1a; $\mathrm{Ar}=\mathrm{H}$

1b; $\mathrm{Ar}=\mathrm{C}_{6} \mathrm{H}_{5}$

1c; $\mathrm{Ar}=2,4,6-\mathrm{Me}_{3} \mathrm{C}_{6} \mathrm{H}_{2}$

1d: $\mathrm{Ar}=4-\mathrm{MeOC}_{6} \mathrm{H}_{4}$

1e; $\mathrm{Ar}=4-\mathrm{NO}_{2} \mathrm{C}_{6} \mathrm{H}_{4}$

Scheme 5. Preparation of the Phosphoric Acids (Yields refer to 1e)

Table 4. Catalytic Enantioselective Mannich-type reactions ${ }^{\mathrm{a}}$

\begin{tabular}{lllll}
\hline Entry & $\mathrm{R}$ & Product & Yield/\% & \%ee \\
\hline 1 & $\mathrm{Ph}$ & $\mathbf{4 a}$ & 98 & 89 \\
2 & $p-\mathrm{CH}_{3} \mathrm{C}_{6} \mathrm{H}_{4}$ & $\mathbf{4 b}$ & 100 & 89 \\
3 & $p-\mathrm{FC}_{6} \mathrm{H}_{4}$ & $\mathbf{4 c}$ & 100 & 85 \\
4 & $p-\mathrm{ClC}_{6} \mathrm{H}_{4}$ & $\mathbf{4 d}$ & 100 & 80 \\
5 & $p-\mathrm{MeOC}_{6} \mathrm{H}_{4}$ & $\mathbf{4 e}$ & 86 & 75 \\
6 & $2-F u r y l$ & $\mathbf{4 f}$ & 88 & 75 \\
\hline
\end{tabular}

${ }^{a}$ Aldimine 2 (1.0 equiv) and 3 (1.5 equiv) were treated with 1 e (10 mol\%) in toluene at $-78{ }^{\circ} \mathrm{C}$ for $24 \mathrm{~h}$. 


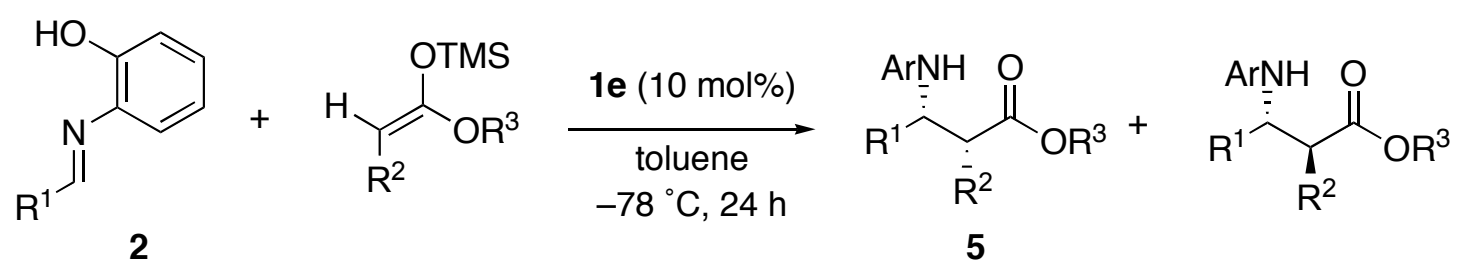

Table 5. Diastereoselective Mannich-type reaction ${ }^{\mathrm{a}}$

\begin{tabular}{|c|c|c|c|c|c|c|c|}
\hline Entry & $\mathrm{R}^{1}$ & $\mathrm{R}^{2}$ & $\mathrm{R}^{3}$ & $\begin{array}{l}\text { Produ } \\
\text { ct }\end{array}$ & $\begin{array}{l}\text { Yiel } \\
\mathrm{d} / \%\end{array}$ & syn/anti & $\% \mathrm{ee}^{\mathrm{b}}$ \\
\hline 1 & $\mathrm{Ph}$ & $\mathrm{Me}^{\mathrm{c}}$ & Et & $5 a$ & 100 & $87: 13$ & 96 \\
\hline 2 & $p-\mathrm{MeOC}_{6} \mathrm{H}_{4}$ & $\mathrm{Me}^{\mathrm{c}}$ & $\mathrm{Et}$ & $5 b$ & 100 & $92: 8$ & 88 \\
\hline 3 & $p-\mathrm{FC}_{6} \mathrm{H}_{4}$ & $\mathrm{Me}^{\mathrm{c}}$ & Et & $5 c$ & 100 & 91:9 & 84 \\
\hline 4 & $p-\mathrm{ClC}_{6} \mathrm{H}_{4}$ & $\mathrm{Me}^{\mathrm{c}}$ & Et & $5 d$ & 100 & $86: 14$ & 83 \\
\hline 5 & $p-\mathrm{MeC}_{6} \mathrm{H}_{4}$ & $\mathrm{Me}^{\mathrm{c}}$ & Et & $5 e$ & 100 & $94: 6$ & 88 \\
\hline 6 & 2-thienyl & $\mathrm{Me}^{\mathrm{c}}$ & Et & $5 f$ & 81 & $94: 6$ & 88 \\
\hline 7 & $\mathrm{PhCH}=\mathrm{CH}$ & $\mathrm{Me}^{\mathrm{c}}$ & $\mathrm{Et}$ & $5 g$ & 91 & $95: 5$ & 90 \\
\hline 8 & $\mathrm{Ph}$ & $\mathrm{PhCH}_{2}{ }^{\mathrm{d}}$ & $\mathrm{Et}$ & $5 \mathrm{~h}$ & 100 & $93: 7$ & 91 \\
\hline 9 & $p-\mathrm{MeOC}_{6} \mathrm{H}_{4}$ & $\mathrm{PhCH}_{2}{ }^{\mathrm{d}}$ & Et & $5 \mathbf{i}$ & 92 & $93: 7$ & 87 \\
\hline 10 & $\mathrm{PhCH}=\mathrm{CH}$ & $\mathrm{PhCH}_{2}{ }^{\mathrm{d}}$ & Et & $5 \mathbf{j}$ & 90 & $95: 5$ & 90 \\
\hline 11 & $\mathrm{Ph}$ & $\mathrm{Ph}_{3} \mathrm{SiO}^{\mathrm{e}}$ & $\mathrm{Me}$ & $5 \mathbf{k}$ & 79 & 100:0 & 91 \\
\hline 12 & $\mathrm{Ph}$ & $(t-\mathrm{Bu}) \mathrm{Me}_{2} \mathrm{SiO}^{\mathrm{f}}$ & $\mathrm{Me}$ & 51 & 96 & $94: 6$ & 85 \\
\hline
\end{tabular}

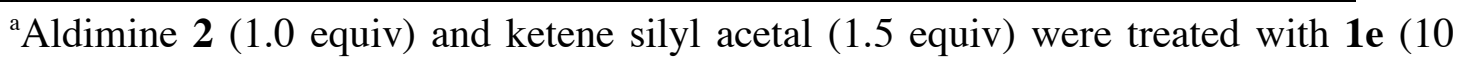
mol\%) in toluene at $-78{ }^{\circ} \mathrm{C}$ for $24 \mathrm{~h}$. ${ }^{\mathrm{b}} \mathrm{Ee}$ of syn isomer. ${ }^{\mathrm{c}} E: Z=87: 13$. ${ }^{\mathrm{d}} E: Z=87: 13$. ${ }^{\mathrm{e}} E: Z=91: 9 .{ }^{\mathrm{f}} E: Z=96: 4$. 
The relative and absolute stereochemistry of the major isomer of $\mathbf{5 k}\left(\mathrm{R}_{1}=\mathrm{Ph}, \mathrm{R}_{2}=\mathrm{Ph}_{3} \mathrm{SiO}\right.$, $\mathrm{R}_{3}=\mathrm{Me}$ ) was unambiguously determined by X-ray analysis (See cif file) and those of others were surmised by the analogy.

\section{Experimental Section}

${ }^{1} \mathrm{H}(400 \mathrm{MHz}),{ }^{13} \mathrm{C}(125 \mathrm{MHz})$, and ${ }^{31} \mathrm{P}(162 \mathrm{MHz})$ NMR spectra were recorded on a Varian Inova 400 spectrometer in $\mathrm{CDCl}_{3}$. Tetramethylsilane (TMS) served as an internal standard $(\delta=0)$ for ${ }^{1} \mathrm{H} \mathrm{NMR}, \mathrm{CDCl}_{3}$ was used as an internal standard $(\delta=77.0)$ for ${ }^{13} \mathrm{C}$ NMR, and $\mathrm{H}_{3} \mathrm{PO}_{4}$ was used as an external standard $(\delta=0)$ for ${ }^{31} \mathrm{P} \mathrm{NMR} . \mathrm{C}_{6} \mathrm{~F}_{6}$ was used as an internal standard $(\delta=0)$ for ${ }^{19} \mathrm{~F}$ NMR. IR spectra were recorded on a Shimadzu FT-IR 8600 spectrometer. EI mass spectra were measured on a Shimadzu GCMS-QP-5000 at an ionizing voltage of $70 \mathrm{eV}$. Fab mass spectra were measured on a JEOL JMS-700 using 3-nitrophenylbenzyl alcohol as a matrix. Purification of the products was performed by column chromatography on silica gel (Fuji sylisia D60L) or preparative TLC on silica gel (Wako gel B-5F). All solvents were purified according to the standard procedures.<smiles>O=[N+]([O-])c1ccc(-c2cc3ccccc3c(-c3c(O)c(-c4ccc([N+](=O)[O-])cc4)cc4ccccc34)c2O)cc1</smiles>

(R)-3,3'-Bis(4-nitrophenyl)-4-yl[1,1']binaphthalenyl-2,2'-diol

This compound was prepared according to the literature procedure. ${ }^{1}$

To a suspension of $(R)-3,3$ '-bis(dihydroxyborane)-2,2'-dimethoxy-1,1'-dinaphthyl ${ }^{1}$ (2.01 g, $5.0 \mathrm{mmol}), \mathrm{Ba}(\mathrm{OH})_{2} \bullet 8 \mathrm{H}_{2} \mathrm{O}(4.75 \mathrm{~g}, 15.1 \mathrm{mmol})$, and $\mathrm{Pd}\left(\mathrm{PPh}_{3}\right)_{4}(430 \mathrm{mg}, 0.372$ 
mmol) in degassed dioxane/water (44 $\mathrm{mL}, 3: 1 / \mathrm{v}: \mathrm{v})$ were added 4-bromonitrobenzene $(2.53 \mathrm{~g}, 12.5 \mathrm{mmol})$. The reaction mixture was heated at reflux for $25 \mathrm{~h}$ and cooled to room temperature. Dioxane was removed, and the resulting residue was redissolved in methylene chloride, washed with $1 \mathrm{~N} \mathrm{HCl}$ solution and brine, dried over anhydrous $\mathrm{Na}_{2} \mathrm{SO}_{4}$, and concentrated in vacuo to give crude products. To the solution of the crude products in $\mathrm{CH}_{2} \mathrm{Cl}_{2}(220 \mathrm{~mL})$ was added a solution of $\mathrm{BBr}_{3}(5.63 \mathrm{~g}, 22.5 \mathrm{mmol})$ in $\mathrm{CH}_{2} \mathrm{Cl}_{2}(20 \mathrm{~mL})$ at $0{ }^{\circ} \mathrm{C}$. After being stirred at room temperature for $9.5 \mathrm{~h}$, the reaction mixture was quenched by dropwise addition of $\mathrm{H}_{2} \mathrm{O}(200 \mathrm{~mL})$ at $0{ }^{\circ} \mathrm{C}$ for 10 min, extracted with $\mathrm{CH}_{2} \mathrm{Cl}_{2}$. The combined organic layers were washed with brine, dried over $\mathrm{Na}_{2} \mathrm{SO}_{4}$, and concentrated in vacuo. Purification by chromatography on $\mathrm{SiO}_{2}($ hexane $/ \mathrm{EtOAc}=5: 1, \mathrm{v} / \mathrm{v})$ gave $2.18 \mathrm{~g}(4.12 \mathrm{mmol})$ of the title compound as a solid in $82 \%$.

$[\alpha]_{\mathrm{D}}{ }^{25}-10.6$ (c 1.0, $\left.\mathrm{CHCl}_{3}\right) ; \mathrm{mp} .215 .5-233.0{ }^{\circ} \mathrm{C}\left(\mathrm{dec}\right.$.); $\quad \mathrm{IR}\left(\mathrm{CHCl}_{3}\right) 3522,3061,3032$, 1622, 1601, 1520, 1348, 853, 793, 777, 773, $665 \mathrm{~cm}^{-1} ; \mathrm{R}_{\mathrm{f}} 0.3$ (Hexane: Ethyl acetate $=5: 1) ;{ }^{1} \mathrm{H}$ NMR $\left(400 \mathrm{MHz}, \mathrm{CDCl}_{3}\right) \delta=8.33-8.29(\mathrm{~m}, 4 \mathrm{H}), 8.10(\mathrm{~s}, 2 \mathrm{H}), 7.98(\mathrm{~d}$, 2H, $J=7.7 \mathrm{~Hz}), 7.95-7.91(\mathrm{~m}, 4 \mathrm{H}), 7.48-7.37$ (m, 4H), 7.22 (d, 2H, J=8.2 Hz), 5.42 (s, $2 \mathrm{H}) ;{ }^{13} \mathrm{C}$ NMR $\left(100 \mathrm{MHz}, \mathrm{CDCl}_{3}\right) \delta=150.0,147.1,144.2,133.3,132.3,130.5,129.4$, $128.9,128.5,125.0,124.0,123.5,111.9 ; \mathrm{MS}(\mathrm{Fab}) \mathrm{m} / \mathrm{z} 529\left([\mathrm{M}+1]^{+}\right), 528\left(\mathrm{M}^{+}\right)$, Found: C, 72.64; H, 3.60; N, 5.37\%. Calcd for $\mathrm{C}_{32} \mathrm{H}_{20} \mathrm{~N}_{2} \mathrm{O}_{6}: \mathrm{C}, 72.72 ; \mathrm{H}, 3.81 ; \mathrm{N}$, $5.30 \%$.<smiles>O=[N+]([O-])c1ccc(-c2cc(-c3c(-c4ccc([N+](=O)[O-])cc4)cc4ccccc4c3OP(=O)(O)O)c3ccccc3c2)cc1</smiles>

(R)-3,3'-bis(4-nitrophenyl)-1,1'-binaphthyl phosphate (1e)

This compound was prepared according to the modified procedure based on the literature procedure. ${ }^{2}$ 
To a solution of $(R)-3,3$ '-bis(4-nitrophenyl)-4-yl[1,1']binaphthalenyl-2,2'-diol (1.01 g, $1.90 \mathrm{mmol})$ in pyridine $(7.8 \mathrm{~mL})$ was added phosphorus oxychloride $(250 \mu \mathrm{L}, 2.68$ mmol) at room temperature. After stirring at room temperature for $3 \mathrm{~h}$, the reaction mixture was quenched by addition of $\mathrm{H}_{2} \mathrm{O}(154 \mu \mathrm{L})$ at $0{ }^{\circ} \mathrm{C}$ and stirred for $1 \mathrm{~h}$ at room temperature. After evaporation of pyridine under vacuum, $6 \mathrm{~N} \mathrm{HCl}(20 \mathrm{~mL})$ was added to the residue at $0{ }^{\circ} \mathrm{C}$. The mixture was refluxed for $2 \mathrm{~h}$. After cooling to $0{ }^{\circ} \mathrm{C}$, the resulting solids were collected by filtration, washed with $\mathrm{H}_{2} \mathrm{O}$ to give crude material $(0.991 \mathrm{~g})$. The crude material was dissolved in $\mathrm{CH}_{2} \mathrm{Cl}_{2}$ and poured into hexane to give 1e as crystals $(0.935 \mathrm{~g}, 1.58 \mathrm{mmol})$ in $83 \%$ yield.

$[\alpha]_{\mathrm{D}}^{25}-34.7$ (c 0.51, EtOH); mp. 223.5-249.0 ${ }^{\circ} \mathrm{C}$ (dec.); $\mathrm{IR}\left(\mathrm{CHCl}_{3}\right)$ 3026, 3017, 1601, 1520, 1501, 1348, 1213, 1016, 961, 851, $669 \mathrm{~cm}^{-1} ;{ }^{1} \mathrm{H}$ NMR (400 MHz, $\left.\mathrm{CDCl}_{3}: \mathrm{CD}_{3} \mathrm{OH}=14: 1 / \mathrm{v}: \mathrm{v}\right) \delta=8.28(\mathrm{~d}, 4 \mathrm{H}, J=8.8 \mathrm{~Hz}), 8.09(\mathrm{~s}, 2 \mathrm{H}), 8.02(\mathrm{~d}, 2 \mathrm{H}, J=8.2$ $\mathrm{Hz}), 7.91(\mathrm{~d}, 4 \mathrm{H}, J=8.8 \mathrm{~Hz}), 7.58-7.54(\mathrm{~m}, 2 \mathrm{H}), 7.41-7.36(\mathrm{~m}, 4 \mathrm{H}) ;{ }^{31} \mathrm{P}$ NMR $(162$ $\left.\mathrm{MHz}, \mathrm{CDCl}_{3}\right) \delta=1.63 ;{ }^{13} \mathrm{C} \mathrm{NMR}\left(100 \mathrm{MHz}, \mathrm{CDCl}_{3}\right) \delta=146.8,145.0,144.5,144.4$, 143.7, 141.1, 132.6, 131.8, 131.4, 131.2, 130.7, 128.6, 127.4, 127.1, 126.4, 123.1, 123.0, 96.1. MS (Fab) m/z $591\left([\mathrm{M}+1]^{+}\right), 590\left(\mathrm{M}^{+}\right)$, Found: C, 65.08; H, 3.53; N, 4.92\%. Calcd for $\mathrm{C}_{32} \mathrm{H}_{19} \mathrm{~N}_{2} \mathrm{O}_{8} \mathrm{P}: \mathrm{C}, 65.09 ; \mathrm{H}, 3.24 ; \mathrm{N}, 4.74 \%$.<smiles>O=P(O)(O)Oc1ccc2ccccc2c1-c1c(O)ccc2ccccc12</smiles>

$(R)$-Binaphthylphosphoric acid(1a) was prepared according to the reported procedure ${ }^{2}$ starting from $(R)$-binaphthol in $56 \%$. $[\alpha]_{\mathrm{D}}^{22}-562.7$ (c 0.97, $\left.\mathrm{CHCl}_{3}\right) ; \mathrm{lit}^{2}[\alpha]_{546}^{22}-113.0$ (c 0.95, $\left.\mathrm{CHCl}_{3}\right)$. IR $\left(\mathrm{CHCl}_{3}\right)$ 3024, $3015,1231,1215,1203,1020,951,797,779,773,762,748,737,723,716,700,673$ $\mathrm{cm}^{-1} .{ }^{1} \mathrm{H} \mathrm{NMR}\left(400 \mathrm{MHz}, \mathrm{CDCl}_{3}\right) \delta=8.01$ (d, $\left.2 \mathrm{H}, J=8.8 \mathrm{~Hz}\right), 7.94(\mathrm{~d}, 2 \mathrm{H}, J=8.2 \mathrm{~Hz})$, 7.55 (d, $2 \mathrm{H}, J=8.8 \mathrm{~Hz}), 7.49-7.44(\mathrm{~m}, 2 \mathrm{H}), 7.38$ (d, $2 \mathrm{H}, J=8.2 \mathrm{~Hz}), 7.32-7.27$ (m, $2 \mathrm{H})$, $3.22(\mathrm{~s}, 1 \mathrm{H}) .{ }^{13} \mathrm{C} \mathrm{NMR}\left(100 \mathrm{MHz}, \mathrm{CDCl}_{3}\right) \delta=147.2,147.1,132.2,131.6,131.0,128.3$, $127.0,126.5,125.5,121.4,120.5$. 
<smiles>Oc1c(-c2ccccc2)cc2ccccc2c1-c1c(O)c(-c2ccccc2)cc2ccccc12</smiles>

(R)-3,3'-Diphenyl-1,1'-binaphthol

was

prepared

from

(R)-2,2'-dimethoxy-3,3'-diphenyl-1,1'-dinaphthol according to the procedure. ${ }^{1}$

$[\alpha]_{\mathrm{D}}^{21}+76.7\left(\right.$ c $\left.1.0, \mathrm{CHCl}_{3}\right) ;$ lit $^{3}[\alpha]_{\mathrm{D}}+69.1\left(\right.$ c $\left.1.00 \mathrm{CHCl}_{3}\right)$. $\mathrm{R}_{\mathrm{f}} 0.5$ (Hexane: Ethyl acetate $=8: 1){ }^{1} \mathrm{H} \mathrm{NMR}\left(400 \mathrm{MHz}, \mathrm{CDCl}_{3}\right) \delta=8.00(\mathrm{~s}, 2 \mathrm{H}), 7.90(\mathrm{~d}, 2 \mathrm{H}, J=8.2 \mathrm{~Hz})$, 7.74-7.71 (m, 4H), 7.51-7.44 (m, 4H), 7.42-7.34 (m, 4H), 7.34-7.27 (m, 2H), 7.25-7.20 $(\mathrm{m}, 2 \mathrm{H}), 5.36(\mathrm{~s}, 2 \mathrm{H}) .{ }^{13} \mathrm{C} \mathrm{NMR}\left(100 \mathrm{MHz}, \mathrm{CDCl}_{3}\right) \delta=151.1,137.4,132.9,131.4$, $130.7,129.6,129.4,128.5,128.4,127.8,127.3,124.3,124.3,112.4$.<smiles>O=P1(O)Oc2cc3ccccc3c(-c3ccccc3)c2-c2c(c(-c3ccccc3)cc3ccccc23)O1</smiles>

(R)-3,3'-Diphenyl-1,1'-binaphyl phosphoric acid (1b) was prepared as the same procedure as that of $(R)$-binaphthylphosphoric acid and recrystallized from ethanol as crystals in $59 \%$ yield.

$[\alpha]_{\mathrm{D}}{ }^{27}-283.5\left(\mathrm{c} 0.99, \mathrm{CHCl}_{3}\right) . \quad 191.0-196.5^{\circ} \mathrm{C}\left(\mathrm{dec}\right.$.). IR $\left(\mathrm{CHCl}_{3}\right) 3059,3011,1499$, 1412, 1269, 1246, 1213, 1182, 1151, 1024, 995, 962, $895 \mathrm{~cm}^{-1}$. ${ }^{1} \mathrm{H}$ NMR (400 MHz, $\left.\mathrm{CDCl}_{3}\right) \delta=7.91-7.82(\mathrm{~m}, 4 \mathrm{H}), 7.64$ (brs, $\left.1 \mathrm{H}\right), 7.59$ (d, 4H, $\left.J=7.5 \mathrm{~Hz}\right), 7.45-7.37(\mathrm{~m}, 2 \mathrm{H})$, $7.34(\mathrm{~d}, 2 \mathrm{H}, J=8.4 \mathrm{~Hz}), 7.27-7.20(\mathrm{~m}, 2 \mathrm{H}), 7.15-7.04(\mathrm{~m}, 4 \mathrm{H}), 7.03-6.96(\mathrm{~m}, 2 \mathrm{H}) .{ }^{31} \mathrm{P}$ 
$\operatorname{NMR}\left(162 \mathrm{MHz}, \mathrm{CDCl}_{3}\right) \delta=1.99 .{ }^{13} \mathrm{C}$ NMR $\left(100 \mathrm{MHz}, \mathrm{CDCl}_{3}\right) \delta=144.4,144.3,136.5$, $133.8,131.6,130.9,129.5,128.0,127.9,127.3,126.7,126.0,125.5,122.2$. Found: $\mathrm{C}$, 76.46; H, 4.52\%. Calcd for $\mathrm{C}_{32} \mathrm{H}_{21} \mathrm{O} 4 \mathrm{P}: \mathrm{C}, 76.79 ; \mathrm{H}, 4.23$.<smiles>Cc1cc(C)c(-c2cc3ccccc3c(-c3c(O)c(-c4c(C)cc(C)cc4C)cc4ccccc34)c2O)c(C)c1</smiles>

(R)-3,3'-Bis(mesityl)-1,1'-binaphthol ${ }^{4}$

$[\alpha]_{\mathrm{D}}^{25}+72.3$ (c 0.97, THF); lit ${ }^{4}[\alpha]_{\mathrm{D}}+70.0$ (c 1.00, THF). $\mathrm{R}_{\mathrm{f}} 0.3$ (Hexane: $\mathrm{CH}_{2} \mathrm{Cl}_{2}=$ 2:1) ${ }^{1} \mathrm{H}$ NMR (400 MHz, $\left.\mathrm{CDCl}_{3}\right) \delta=7.84(\mathrm{~d}, 2 \mathrm{H}, J=8.1 \mathrm{~Hz}), 7.72(\mathrm{~s}, 2 \mathrm{H}), 7.36-7.23$ (m, $6 \mathrm{H}), 6.98(\mathrm{~s}, 4 \mathrm{H}), 5.00(\mathrm{~s}, 2 \mathrm{H}), 2.31(\mathrm{~s}, 6 \mathrm{H}), 2.13$ (s, 6H), 2.06 (s, 6H), 13C NMR (100 $\left.\mathrm{MHz}, \mathrm{CDCl}_{3}\right) \delta=150.0,137.7,137.1,137.0,133.4,132.9,130.6,129.4,129.4,128.5$, 128.4, 128.2, 126.8, 124.5, 123.8, 112.9, 21.1, 20.5, 20.4. Found: C, 87.05; H, 6.57\%. Calcd for $\mathrm{C}_{38} \mathrm{H}_{34} \mathrm{O}_{2}$ : C, 87.32; $\mathrm{H}, 6.56 \%$.<smiles>COc1c(-c2c(C)cc(C)cc2C)cc2ccccc2c1-c1c(OP(=O)(O)O)c(-c2c(C)cc(C)cc2C)cc2ccccc12</smiles>

(R)-3,3'-Bis(mesityl)-1,1'-binaphthyl phosphate (1c) $[\alpha]_{\mathrm{D}}{ }^{26}-93.2\left(\mathrm{c} 1.08, \mathrm{CHCl}_{3}\right)$. dec at about $224.0-230.5{ }^{\circ} \mathrm{C}, \quad \mathrm{IR}\left(\mathrm{CHCl}_{3}\right) 3007,2922$, 1612, 1489, 1437, 1406, 1244, 1196, 1150, 1130, 997, 970, 899, $853 \mathrm{~cm}^{-1} .{ }^{1} \mathrm{H}$ NMR 
$\left(400 \mathrm{MHz}, \mathrm{CDCl}_{3}\right) \delta=7.89$ (d, 2H, $\left.J=8.1 \mathrm{~Hz}\right), 7.73(\mathrm{~s}, 2 \mathrm{H}), 7.46$ (dd, $\left.2 \mathrm{H}, J=8.1,7.5 \mathrm{~Hz}\right)$, 7.36 (d, 2H, $J=8.1 \mathrm{~Hz}$ ), 7.28 (dd, $2 \mathrm{H}, J=8.1,7.5 \mathrm{~Hz}), 6.76$ (s, $2 \mathrm{H}), 6.68$ (s, $2 \mathrm{H}), 2.13$ (s, $6 \mathrm{H}$ ), 2.00 (s, $6 \mathrm{H}), 1.96$ (s, $6 \mathrm{H}) .{ }^{31} \mathrm{P}$ NMR $\left(162 \mathrm{MHz}, \mathrm{CDCl}_{3}\right) \delta=2.37 .{ }^{13} \mathrm{C}$ NMR $\left(75 \mathrm{MHz}, \mathrm{CDCl}_{3}\right) \delta=145.9,145.8,137.2,136.8,136.5,133.7,132.9,132.0,131.2$, $128.3,128.0,127.3,126.9,126.4,125.9,125.2,122.3,21.1,21.0,20.4$. Found: C, 77.99; H, 5.41\%. Calcd for $\mathrm{C}_{38} \mathrm{H}_{33} \mathrm{O}_{4} \mathrm{P}: \mathrm{C}, 78.07 ; \mathrm{H}, 5.69 \%$.

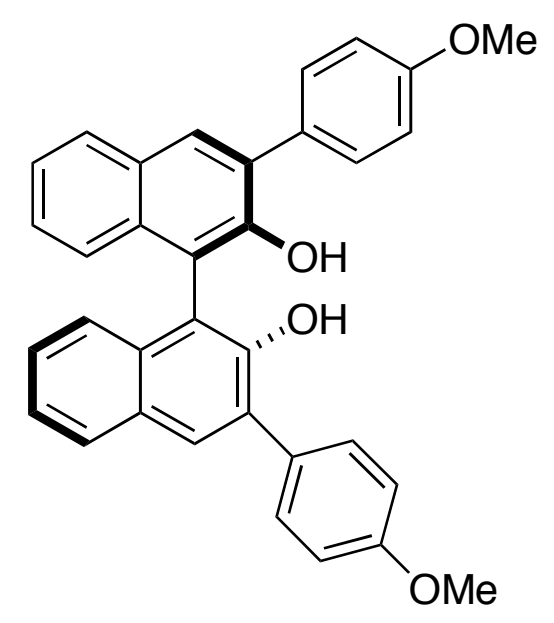

(R)-3,3'-Bis( $p$-methoxyphenyl)-1,1'-binaphthol ${ }^{5}$

$[\alpha]_{\mathrm{D}}{ }^{25}+42.8 \quad\left(\mathrm{c} 1.03, \mathrm{CHCl}_{3}\right) . \quad \mathrm{Mp} 108.5-111.0{ }^{\circ} \mathrm{C}$. (reprecipitated from $\left.\mathrm{CH}_{2} \mathrm{Cl}_{2} / \mathrm{Hexane}\right)$. IR $\left(\mathrm{CHCl}_{3}\right) 3522,3011,2937,2839,1732,1611,1514,1439,1408$, $1385,1362,1283,1248,1178,1148,1128,1036,908,833 \mathrm{~cm}^{-1} . \mathrm{R}_{\mathrm{f}} 0.2$ (Hexane: Ethyl acetate $=5: 1) \quad{ }^{1} \mathrm{H}$ NMR $\left(400 \mathrm{MHz}, \mathrm{CDCl}_{3}\right) \delta=7.98(\mathrm{~s}, 2 \mathrm{H}), 7.90(\mathrm{~d}, 2 \mathrm{H}, J=8.1 \mathrm{~Hz})$, 7.67 (d, 4H, $J=9.0 \mathrm{~Hz}), 7.40-7.34(\mathrm{~m}, 2 \mathrm{H}), 7.32-7.27(\mathrm{~m}, 2 \mathrm{H}), 7.21(\mathrm{~d}, 2 \mathrm{H}, J=9.0 \mathrm{~Hz})$, 7.01 (d, 4H, $J=9.0 \mathrm{~Hz}), 5.37$ (s, 2H), 3.85 (s, $\left.6 \mathrm{H}) .{ }^{13} \mathrm{C} \mathrm{NMR} \mathrm{(100} \mathrm{MHz,} \mathrm{CDCl}_{3}\right) \delta=159.3$, 150.2, 132.8, 130.9, 130.7, 130.3, 129.8, 129.5, 128.3, 127.1, 124.3, 124.2, 114.0, 112.4, 55.3. Found: $\mathrm{C}, 82.01 ; \mathrm{H}, 5.43 \%$. Calcd for $\mathrm{C}_{34} \mathrm{H}_{26} \mathrm{O}_{4}: \mathrm{C}, 81.91 ; \mathrm{H}, 5.26 \%$. 
<smiles>COc1ccc(-c2cc3ccccc3c3c2Oc2c(-c4ccc(OC)cc4)cc4ccccc4c2-c2c-3c(-c3ccc(OC)cc3)cc3ccccc23)cc1</smiles>

(R)-3,3'-Bis(p-methoxyphenyl)-1,1'-binaphthyl phosphate (1d)

$[\alpha]_{\mathrm{D}}{ }^{26}-145.0\left(\mathrm{c} 0.98, \mathrm{CH}_{2} \mathrm{Cl}_{2}\right) . \quad \mathrm{mp} 194.0-200.5{ }^{\circ} \mathrm{C}$ (dec.). IR $\left(\mathrm{CHCl}_{3}\right) 3009,2937$, $2839,1611,1516,1427,1408,1286,1248,1178,1151,1034,997,962,897,887,833$ $\mathrm{cm}^{-1} . \quad{ }^{1} \mathrm{H}$ NMR $\left(400 \mathrm{MHz}, \mathrm{CDCl}_{3}\right) \delta=7.99$ (s, $\left.2 \mathrm{H}\right), 7.94$ (d, $\left.2 \mathrm{H}, J=8.1 \mathrm{~Hz}\right), 7.55(\mathrm{~d}, 4$ $\mathrm{H}, J=8.7 \mathrm{~Hz}$ ), 7.51-7.46 (m, $2 \mathrm{H}), 7.36-7.26$ (m, $4 \mathrm{H}), 6.84$ (d, $4 \mathrm{H}, J=8.7 \mathrm{~Hz}), 5.31$ (brs, $1 \mathrm{H}), 3.54(\mathrm{~s}, 6 \mathrm{H}) . \quad{ }^{31} \mathrm{P} \mathrm{NMR}\left(162 \mathrm{MHz}, \mathrm{CDCl}_{3}\right) \delta=3.50 . \quad{ }^{13} \mathrm{C} \mathrm{NMR}(100 \mathrm{MHz}$, $\left.\mathrm{CDCl}_{3}\right) \delta=159.0,144.5,144.3,133.5,133.4,131.6,131.5,130.9,130.8,128.8,128.2$, 126.9, 126.2, 125.8, 122.3, 113.7, 55.1. Found: C, 72.60; H, 4.55\%. Calcd for $\mathrm{C}_{34} \mathrm{H}_{25} \mathrm{O}_{6} \mathrm{P}: \mathrm{C}, 72.85 ; \mathrm{H}, 4.50 \%$.

General Procedure for the enantioselective Mannich-type reaction for the preparation of 3a (Table 2, Entry 1).

To a solution of $N$-benzylidene-2-hydroxyaniline $\mathbf{2 a}(31.2 \mathrm{mg}, 0.158 \mathrm{mmol})$ and a phosphate $1 \mathrm{~d}(10.0 \mathrm{mg}, 0.0169 \mathrm{mmol})$ in toluene $(1 \mathrm{~mL})$ at $-78{ }^{\circ} \mathrm{C}$ was added dropwise a solution of a ketene silyl acetal $1(50 \mu \mathrm{L}, 0.234 \mathrm{mmol})$ for $3 \mathrm{~min}$. After being stirred at the temperature for $13 \mathrm{~h}$, the mixture was quenched by addition of sat. $\mathrm{NaHCO}_{3}$ and sat. KF solution at $-78{ }^{\circ} \mathrm{C}$. After filtration over Celite, the filtrate was extracted with ethyl acetate. The combined organic layers were successively washed with $1 \mathrm{H} \mathrm{HCl}$, brine, dried over anhydrous $\mathrm{Na}_{2} \mathrm{SO}_{4}$, and concentrated to dryness. The remaining solid was purified by TLC $\left(\mathrm{SiO}_{2}\right.$, hexane : ethyl acetate $\left.=3: 1 / \mathrm{v}: \mathrm{v}\right)$ to give a $\beta$-amino ester $4 \mathbf{a}$ (45.6 $\mathrm{mg}, 0.155 \mathrm{mmol}$ ) in $98 \%$ yield. The enantiomeric excess was determined by Daicel Chiralpak AD-H column. 


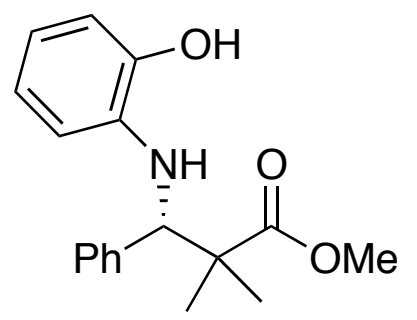

Methyl 3-(N-2-hydroxyphenylamino)-2,2-dimethyl-3-phenylpropionate (4a) $)^{7,8}$ $[\alpha]_{\mathrm{D}}{ }^{25}+0.2\left(\mathrm{c} 1.03, \mathrm{CHCl}_{3}\right)\left(89 \%\right.$ ee); $\mathrm{R}_{\mathrm{f}} 0.4$ (Hexane: Ethyl acetate=3:1); ${ }^{1} \mathrm{H}$ NMR $\left(400 \mathrm{MHz}, \mathrm{CDCl}_{3}\right) \delta=7.29-7.19(\mathrm{~m}, 5 \mathrm{H}), 6.69(1 \mathrm{H}, \mathrm{dd}, J=7.7,1.5 \mathrm{~Hz}), 6.61(1 \mathrm{H}, \mathrm{ddd}$, $J=7.7,7.7,1.5 \mathrm{~Hz}), 6.53(1 \mathrm{H}, \mathrm{ddd}, J=7.7,7.7,1.5 \mathrm{~Hz}), 6.38(1 \mathrm{H}, \mathrm{dd}, J=7.7,1.5 \mathrm{~Hz})$, 5.80 (brs, 1H), 4.55 (brs, 1H), 4.55 (s, $1 \mathrm{H}), 3.69$ (s, 3H), 1.24 (s, 3H), 1.22 (s, 3H); ${ }^{13} \mathrm{C} \mathrm{NMR}\left(100 \mathrm{MHz}, \mathrm{CDCl}_{3}\right) \delta=177.7,144.3,139.0,135.5,128.3,127.9,127.41,121.0$, 117.9, 114.4, 113.9, 64.6, 52.2, 47.4, 24.4, 20.0; HPLC: Daicel Chiralpak AD-H, Hexane $/ i-\mathrm{PrOH}=5 / 1$, Flow rate $0.5 \mathrm{~mL} / \mathrm{min}, \mathrm{UV}=244 \mathrm{~nm}, \mathrm{t}_{\mathrm{R}}=11.1 \mathrm{~min}$ (minor isomer, $3 R$ ), $\mathrm{t}_{\mathrm{R}}=16.3 \min$ (major isomer, $3 S$ ).<smiles>COC(=O)C(C)(C)[C@H](Nc1ccccc1O)c1ccc(C)cc1</smiles>

Methyl 3-(N-2-hydroxyphenylamino)-2,2-dimethyl-3-(4-methylphenyl)propionate (4b) $[\alpha]_{\mathrm{D}}{ }^{24}+16.2\left(\mathrm{c} 0.99, \mathrm{CHCl}_{3}\right)(89 \%$ ee $) ; \quad$ oil; $\operatorname{IR}\left(\mathrm{CHCl}_{3}\right) 3603,3425,3007,1724$, 1611, 1514, 1448, 1229, 1140, 791, $775 \mathrm{~cm}^{-1} ; \mathrm{R}_{\mathrm{f}} 0.3$ (Hexane: Ethyl acetate=3:1); ${ }^{1} \mathrm{H}$ NMR $\left(400 \mathrm{MHz}, \mathrm{CDCl}_{3}\right) \delta=7.16(\mathrm{~d}, 2 \mathrm{H}, J=8.1 \mathrm{~Hz}), 7.07(\mathrm{~d}, 2 \mathrm{H}, J=8.1 \mathrm{~Hz}), 6.69$ (dd, 1H, $J=7.6,1.1 \mathrm{~Hz}), 6.60$ (ddd, $1 \mathrm{H}, J=7.6,7.6,1.1 \mathrm{~Hz}$ ), 6.51 (ddd, 1H, $J=7.6,7.6$, $1.3 \mathrm{~Hz}$ ), 6.39 (dd, 1H, J=7.6, $1.3 \mathrm{~Hz}$ ), 5.98 (brs, 1H), 4.90 (brs, 1H), 4.54 (s, 1H), 3.68 $(\mathrm{s}, 3 \mathrm{H}), 2.28(\mathrm{~s}, 3 \mathrm{H}), 1.23(\mathrm{~s}, 3 \mathrm{H}), 1.21(\mathrm{~s}, 3 \mathrm{H}) ;{ }^{13} \mathrm{C} \mathrm{NMR}\left(100 \mathrm{MHz}, \mathrm{CDCl}_{3}\right) \delta=$ $177.8,144.3,136.9,135.9,135.7,128.7,128.2,121.0,117.9114 .3,113.9,64.3,52.2$, 47.4, 24.3, 21.0, 20.0. MS (EI) m/z 313(M+1, 1), 213(15), 212(100), 211(16), 210(25), 120(24), 105(12), 90(14), 73(8), 65(14); Found: C, 72.98; H, 7.54; N, 4.53\%. Calcd for $\mathrm{C}_{19} \mathrm{H}_{23} \mathrm{NO}_{3}$ : C, 72.82; $\mathrm{H}, 7.40 ; \mathrm{N}, 4.47 \%$. HPLC: Daicel Chiralpak AD-H, 
Hexane $/ i-\mathrm{PrOH}=5 / 1$, Flow rate $0.6 \mathrm{~mL} / \mathrm{min}, \mathrm{UV}=244 \mathrm{~nm}, \mathrm{t}_{\mathrm{R}}=8.3 \mathrm{~min}$ (minor isomer, $3 R$ ), $\mathrm{t}_{\mathrm{R}}=13.6 \mathrm{~min}$ (major isomer, $3 S$ ).<smiles>COC(=O)C(C)(C)[C@H](Nc1ccccc1O)c1ccc(F)cc1</smiles>

Methyl 3-(4-fluorophenyl)-3-( $N$-2-hydroxyphenylamino)-2,2-dimethylpropionate (4c) $[\alpha]_{\mathrm{D}}{ }^{24}-15.4\left(\mathrm{c} 1.06, \mathrm{CHCl}_{3}\right)\left(85 \%\right.$ ee); ; oil; IR $\left(\mathrm{CHCl}_{3}\right) 3601,3422,3007,2361$, 1724, 1609, 1510, 1448, 1265, 1232, 1153, 1140, $841 \mathrm{~cm}^{-1} ; \mathrm{R}_{\mathrm{f}} 0.3$ (Hexane: Ethyl acetate $=3: 1) ; \quad{ }^{1} \mathrm{H}$ NMR $\left(400 \mathrm{MHz}, \mathrm{CDCl}_{3}\right) \delta=7.25(\mathrm{dd}, 2 \mathrm{H}, J=8.6,7.0 \mathrm{~Hz}), 7.00(\mathrm{dd}$, 2H, $J=8.6,8.6 \mathrm{~Hz}$ ), 6.69 (dd, 1H, J=7.7, $1.5 \mathrm{~Hz}), 6.62$ (dd, 1H, J=7.7, 7.7 Hz), 6.53 (dd, $1 \mathrm{H}, J=7.7,7.7 \mathrm{~Hz}$ ), 6.34 (dd, 1H, $J=7.7,1.5 \mathrm{~Hz}$ ), 5.56 (brs, 1H), 4.93 (brs, 1H), 4.54 (s, $1 \mathrm{H}), 3.69(\mathrm{~s}, 3 \mathrm{H}), 1.24(\mathrm{~s}, 3 \mathrm{H}), 1.20(\mathrm{~s}, 3 \mathrm{H}) ;{ }^{13} \mathrm{C} \mathrm{NMR}\left(100 \mathrm{MHz}, \mathrm{CDCl}_{3}\right) \delta=177.4$, $163.3,160.9,144.1,135.4,134.8,129.8\left(\mathrm{~d}, \mathrm{~J}_{\mathrm{F}-\mathrm{C}}=8.0 \mathrm{~Hz}\right), 121.1,117.9,114.9\left(\mathrm{~d}, \mathrm{~J}_{\mathrm{F}-\mathrm{C}}=\right.$ $8.0 \mathrm{~Hz}), 114.3,113.6,63.9,52.3,47.3,24.2,20.2$; ${ }^{19} \mathrm{~F}$ NMR $\left(376 \mathrm{MHz}, \mathrm{CDCl}_{3}\right) \delta=$ -46.52; MS (EI) m/z 317(M+, 2), 217(15), 216(100), 215(16), 214(24), 120(22), 109(15), 93(11), 73(6), 65(13); Found: C, 68.04; H, 6.50; N, 4.40\%. Calcd for $\mathrm{C}_{18} \mathrm{H}_{20} \mathrm{FNO}_{3}$ : C, 68.12; H, 6.35; N, 4.41\%. HPLC: Daicel Chiralpak AD-H, Hexane $/ i-\mathrm{PrOH}=5 / 1$, Flow rate $0.5 \mathrm{~mL} / \mathrm{min}, \mathrm{UV}=244 \mathrm{~nm}, \mathrm{t}_{\mathrm{R}}=10.5 \mathrm{~min}$ (minor isomer, $3 R$ ), $\mathrm{t}_{\mathrm{R}}=14.9 \min$ (major isomer, $3 S$ ).<smiles>COC(=O)C(C)(C)[C@H](Nc1ccccc1O)c1ccc(Cl)cc1</smiles>

Methyl 3-(4-chlorophenyl)-3-( $N$-2-hydroxyphenylamino)-2,2-dimethylpropionate (4d) ${ }^{7}$ $[\alpha]_{\mathrm{D}}^{25}+7.8\left(\mathrm{c} 0.99, \mathrm{CHCl}_{3}\right)(80 \%$ ee $)$, lit. $(R \text {-isomer })^{7}[\alpha]_{\mathrm{D}}{ }^{24}-6.5\left(\mathrm{c} 1.60, \mathrm{CHCl}_{3}\right) ; \quad \mathrm{R}_{\mathrm{f}}$ 0.3 (Hexane: Ethyl acetate=3:1); ${ }^{1} \mathrm{H}$ NMR $\left(400 \mathrm{MHz}, \mathrm{CDCl}_{3}\right) \delta=7.26-7.21(\mathrm{~m}, 4 \mathrm{H})$, 
$6.69(\mathrm{dd}, 1 \mathrm{H}, J=7.7,1.5 \mathrm{~Hz}), 6.62(\mathrm{ddd}, 1 \mathrm{H}, J=7.7,7.7,1.5 \mathrm{~Hz}), 6.53$ (ddd, 1H, $J=7.7$, 7.7, $1.5 \mathrm{~Hz}), 6.32$ (dd, 1H, J=7.7, $1.5 \mathrm{~Hz}), 5.80$ (brs, 1H), 4.97 (brs, 1H), 4.54 (s, 1H), $3.68(\mathrm{~s}, 3 \mathrm{H}), 1.24(\mathrm{~s}, 3 \mathrm{H}), 1.20(\mathrm{~s}, 3 \mathrm{H}) ;{ }^{13} \mathrm{C} \mathrm{NMR}\left(100 \mathrm{MHz}, \mathrm{CDCl}_{3}\right) \delta=177.4,144.0$, $137.7,135.3,133.2,129.7,128.2,121.1,117.9,114.2,113.5,64.0,52.3,47.2,24.2$, 20.2. HPLC: Daicel Chiralpak AD-H, Hexane $/ i-\mathrm{PrOH}=5 / 1$, Flow rate $0.5 \mathrm{~mL} / \mathrm{min}$, $\mathrm{UV}=244 \mathrm{~nm}, \mathrm{t}_{\mathrm{R}}=10.2 \mathrm{~min}$ (minor isomer, $3 R$ ), $\mathrm{t}_{\mathrm{R}}=15.7 \mathrm{~min}$ (major isomer, $3 S$ ).

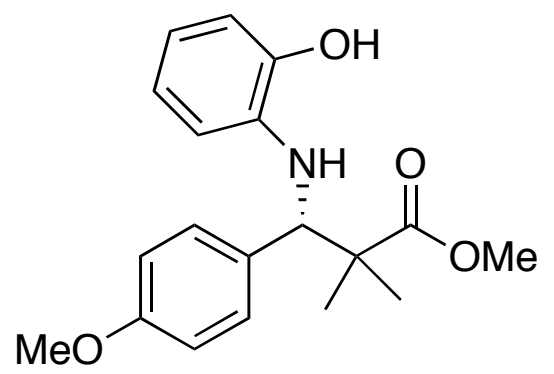

Methyl 3-(N-2-hydroxyphenylamino)-3-(4-methoxyphenyl)-2,2-dimethylpropionate (4e)

$[\alpha]_{\mathrm{D}}{ }^{24}+8.9\left(\mathrm{c} 0.99, \mathrm{CHCl}_{3}\right)(75 \%$ ee $) ; \quad$ oil; $\quad$ IR $\left(\mathrm{CHCl}_{3}\right) 3690,3425,3007,1724,1611$, 1512, 1466, 1445, 1245, 1177, 1140, 1107, 1036, $837 \mathrm{~cm}^{-1}$; $\mathrm{R}_{\mathrm{f}} 0.3$ (Hexane: Ethyl acetate $=3: 1) ; \quad{ }^{1} \mathrm{H}$ NMR $\left(400 \mathrm{MHz}, \mathrm{CDCl}_{3}\right) \delta=7.19(\mathrm{~d}, 2 \mathrm{H}, J=8.7 \mathrm{~Hz}), 6.80(\mathrm{~d}, 2 \mathrm{H}$, $J=8.7 \mathrm{~Hz}), 6.68(\mathrm{dd}, 1 \mathrm{H}, J=7.6,1.2 \mathrm{~Hz}), 6.61(\mathrm{ddd}, 1 \mathrm{H}, J=7.6,7.6,1.2 \mathrm{~Hz}), 6.52$ (ddd, $1 \mathrm{H}, J=7.6,7.6,1.2 \mathrm{~Hz}), 6.38$ (dd, 1H, $J=7.6,1.2 \mathrm{~Hz}), 5.77$ (brs, 1H), 4.87 (brs, 1H), $4.52(\mathrm{~s}, 1 \mathrm{H}), 3.75(\mathrm{~s}, 3 \mathrm{H}), 3.68(\mathrm{~s}, 3 \mathrm{H}), 1.23(\mathrm{~s}, 3 \mathrm{H}), 1.20(\mathrm{~s}, 3 \mathrm{H}) ;{ }^{13} \mathrm{C} \mathrm{NMR}(100 \mathrm{MHz}$, $\left.\mathrm{CDCl}_{3}\right) \delta=177.8,158.8,144.3,135.6,130.9,129.3,121.0,117.9,114.2,114.1,113.3$, 64.0, 55.1, 52.2, 47.4, 24.3, 20.0. MS (EI) m/z 329(M+, 5), 229(20), 228(100), 226(28), 207(38), 182(11), 135(12), 121(15), 120(15), 77(11), 73(13), 69(11), 59(13), 51(10). Found: C, 69.42; H, 6.92; N, 4.23\%. Calcd for $\mathrm{C}_{19} \mathrm{H}_{23} \mathrm{NO}_{4}$ : C, 69.28; H, 7.04; N, 4.25\%. HPLC: Daicel Chiralpak AD-H, Hexane $/ i-\mathrm{PrOH}=5 / 1$, Flow rate $0.7 \mathrm{~mL} / \mathrm{min}$, $\mathrm{UV}=244 \mathrm{~nm}, \mathrm{t}_{\mathrm{R}}=8.9 \mathrm{~min}$ (minor isomer, $3 R$ ), $\mathrm{t}_{\mathrm{R}}=16.8 \mathrm{~min}$ (major isomer, $3 S$ ). 


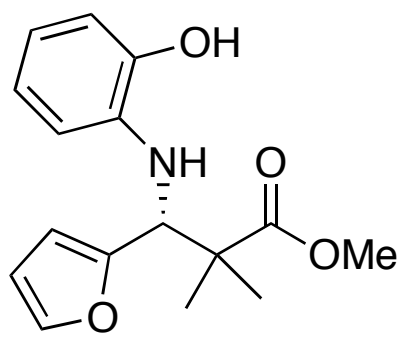

Methyl -3-(2-furyl)-3-( $N$-2-hydroxyphenylamino)-2,2-dimethylpropionate (4f) $[\alpha]_{\mathrm{D}}{ }^{24}+52.2$ (c $\left.0.92, \mathrm{CHCl}_{3}\right)\left(75 \%\right.$ ee); amorphous solid; IR $\left(\mathrm{CHCl}_{3}\right) 3601,3356$, 3013, 1722, 1611, 1502, 1450, 1267, 1221, 1148, $1013 \mathrm{~cm}^{-1} ; \quad \mathrm{R}_{\mathrm{f}} 0.4$ (Hexane: Ethyl acetate $=5: 1) ; \quad{ }^{1} \mathrm{H}$ NMR $\left(400 \mathrm{MHz}, \mathrm{CDCl}_{3}\right) \delta=7.35(\mathrm{dd}, 1 \mathrm{H}, J=1.8,0.8 \mathrm{~Hz}), 6.77(\mathrm{dd}$, $1 \mathrm{H}, J=7.5,10.6 \mathrm{~Hz}$ ), $6.72(\mathrm{ddd}, 1 \mathrm{H}, J=7.5,7.5,1.6 \mathrm{~Hz}$ ), 6.67 (ddd, $1 \mathrm{H}, J=7.5,7.5,1.6$ $\mathrm{Hz}), 6.54$ (dd, $1 \mathrm{H}, J=7.5,1.6 \mathrm{~Hz}), 6.30$ (brs, 1H), 6.26 (dd, 1H, $J=3.3,1.8 \mathrm{~Hz}), 6.12$ (dd, $1 \mathrm{H}, J=3.3,0.8 \mathrm{~Hz}), 4.57$ (s, 1H), 4.22 (brs, 1H), 3.74 (s, 1H), 1.33 (s, 3H), 1.21 (s, 3H); ${ }^{13} \mathrm{C}$ NMR $\left(100 \mathrm{MHz}, \mathrm{CDCl}_{3}\right) \delta=177.9,152.8,147.2,141.8,134.3,121.3,120.5,118.1$, 114.9, 110.1, 108.8, 60.0, 52.4, 47.4, 24.1, 19.9; MS (EI) m/z 289(M+, 5), 207(13), 189(18), 188(100), 186(29), 185(13), 120(11), 81(19), 77(18), 73(11), 59(10), 51(11); Found: C, 66.66; H, 6.52; N, 4.91\%. Calcd for $\mathrm{C}_{16} \mathrm{H}_{19} \mathrm{NO}_{4}: \mathrm{C}, 66.42 ; \mathrm{H}, 6.62 ; \mathrm{N}$, 4.84\%. HPLC: Daicel Chiralpak AD-H, Hexane $/ i-\mathrm{PrOH}=15 / 1$, Flow rate $0.5 \mathrm{~mL} / \mathrm{min}$, $\mathrm{UV}=244 \mathrm{~nm}, \mathrm{t}_{\mathrm{R}}=22.9 \mathrm{~min}$ (minor isomer, $3 R$ ), $\mathrm{t}_{\mathrm{R}}=26.7 \mathrm{~min}$ (major isomer, $3 S$ ).

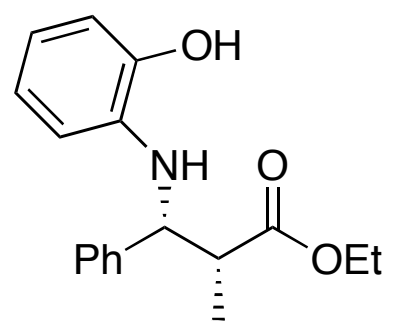

Ethyl 2-methyl-3- $N$-(2-hydroxyphenyl)amino-3-phenylpropionate (5a) (syn/anti=87/13) IR $\left(\mathrm{CHCl}_{3}\right)$ 3603, 3342, 3028, 2986, 1724, 1611, 1514, 1497, 1454, 1267, 1202, 1184 $\mathrm{cm}^{-1} ; \quad \mathrm{R}_{\mathrm{f}}$ 0.2(Hexane: Ethyl acetate $\left.=3: 1\right) ;{ }^{1} \mathrm{H}$ NMR $\left(400 \mathrm{MHz}, \mathrm{CDCl}_{3}\right) \delta=7.35-7.20(\mathrm{~m}$, $5 \mathrm{H}), 6.76-6.53$ (m, 3H), 6.42 (dd, 1H, $J=7.9,1.5 \mathrm{~Hz}, \mathrm{syn}), 6.33$ (dd, $1 \mathrm{H}, J=7.8,1.5 \mathrm{~Hz}$, anti), 6.00 (brs, 1H, anti), 5.46 (brs, 1H, syn), 4.76 (brs, 1H, syn), 4.72 (d, $1 \mathrm{H}, J=4.8 \mathrm{~Hz}$, syn), 4.34 (brs, 1H, anti), 4.33 (d, 1H, J=8.8 Hz, anti), 4.17 (q, $2 \mathrm{H}, J=7.1 \mathrm{~Hz}$, anti), 4.06 (q, 2H, J=7.1 Hz, syn), 2.96 (dq, 1H, J=4.8 Hz, 7.1 Hz, syn), 2.89 (dq, 1H, J=8.8 $\mathrm{Hz}, 7.1 \mathrm{~Hz}$, anti), 1.24 (t, $3 \mathrm{H}, J=7.1 \mathrm{~Hz}$, anti), 1.21 (d, 3H, $J=7.1 \mathrm{~Hz}$, syn), 1.14 (t, 3H, $J=7.1 \mathrm{~Hz}, s y n), 1.09\left(\mathrm{~d}, 3 \mathrm{H}, J=7.1 \mathrm{~Hz}\right.$, anti); ${ }^{13} \mathrm{C} \mathrm{NMR}\left(100 \mathrm{MHz}, \mathrm{CDCl}_{3}\right) \delta=176.1$ 
(anti), 174.6 (syn), 145.8 (anti), 143.9 (syn), 140.8 (syn), 135.8 (syn), 134.8 (anti), 128.5, 128.4, 128.1, 127.5, 127.3, 127.0, 126.9, 121.1 (syn), 120.6 (anti), 119.6 (anti), 117.7 (syn), 116.1 (anti), 114.6 (anti), 114.3 (anti), 113.4 (syn), 62.1 (anti), 61.0 (anti), 60.9 (syn), 60.0 (syn), 46.8 (anti), 46.4 (anti), 15.3 (anti), 14.1 (anti), 13.9 (syn), 12.0 (syn); MS(DI) m/z 299(M+1, 6), 198(100), 135(7), 120(14), 117(9), 115(6), 105(10), 91(24), 77(17), 65(14); Found: C, 72.37; H, 7.29; N, 4.56\%. Calcd for $\mathrm{C}_{18} \mathrm{H}_{21} \mathrm{NO}_{3}$ : C, 72.22; H, 7.07; N, 4.68\%. HPLC: Daicel Chiralpak AS-H, Hexane $/ i-\mathrm{PrOH}=30 / 1$, Flow rate $0.55 \mathrm{~mL} / \mathrm{min}, \mathrm{UV}=244 \mathrm{~nm}, \mathrm{t}_{\mathrm{R}}=48.3 \mathrm{~min}$ (major isomer $, 2 R, 3 R$ ), $\mathrm{t}_{\mathrm{R}}=56.7$ min (minor isomer, $2 S, 3 S)$.

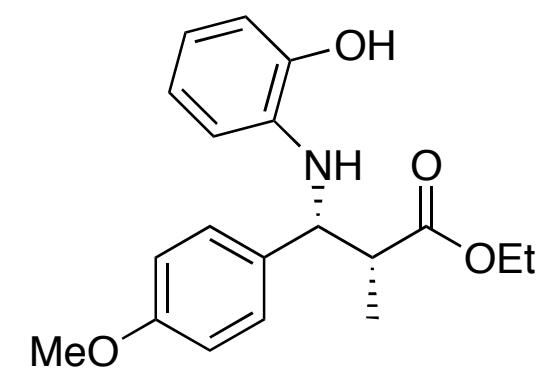

Ethyl 3-N-(2-hydroxyphenyl)amino-3-(4-methoxyphenyl)-2-methylpropionate $($ syn/anti $=92 / 8)$

IR $\left(\mathrm{CHCl}_{3}\right)$ 3603, 3422, 3013, 2982, 2937, 1724, 1611, 1512, 1448, 1375, 1250, 1178, 1109, 1036, $829 \mathrm{~cm}^{-1}$. $\mathrm{R}_{\mathrm{f}} 0.2$ (Hexane: Ethyl acetate=3:1), ${ }^{1} \mathrm{H}$ NMR (300 MHz, $\left.\mathrm{CDCl}_{3}\right) \delta=7.23(\mathrm{~d}, 2 \mathrm{H}, J=8.6 \mathrm{~Hz}, \mathrm{syn}), 7.15$ (d, 2H, $J=8.6 \mathrm{~Hz}$, anti), 6.83 (d, 2H, $J=8.6$ $\mathrm{Hz}), 6.76-6.53$ (m, 3H), 6.43 (dd, 1H, J=7.9, $1.4 \mathrm{~Hz}, \mathrm{syn}), 6.36$ (dd, 1H, J=7.3, $1.8 \mathrm{~Hz}$, anti), 5.95 (brs, 1H, anti), 5.31 (brs, 1H, syn), 4.66 (d, $1 \mathrm{H}, J=5.5 \mathrm{~Hz}$, syn), 4.29 (d, $1 \mathrm{H}$, $J=9.2 \mathrm{~Hz}$, anti), 4.20 (q, $2 \mathrm{H}, J=7.1 \mathrm{~Hz}$, anti), 4.07 (q, $2 \mathrm{H}, J=7.2 \mathrm{~Hz}, s y n), 3.77$ (s, 3H, syn), 3.77 (s, 3H, anti), 2.95 (dq, $1 \mathrm{H}, J=7.1,5.5 \mathrm{~Hz}$, syn), 2.85 (dq, $1 \mathrm{H}, J=9.2 \mathrm{~Hz}, 7.1$ $\mathrm{Hz}$, anti), 1.26 (t, $3 \mathrm{H}, J=7.1 \mathrm{~Hz}$, anti), 1.22 (d, $3 \mathrm{H}, J=7.1 \mathrm{~Hz}$, syn), 1.16 (t, $3 \mathrm{H}, J=7.2$ $\mathrm{Hz}, s y n), 1.09$ (d, $3 \mathrm{H}, J=7.1 \mathrm{~Hz}$, anti). 13C NMR (75 MHz, $\mathrm{CDCl}_{3}$ ) $\delta=176.0$ (anti), 174.4 (syn), 158.7 (anti), 158.5 (syn), 146.1 (anti), 143.9 (anti), 135.7 (syn), 134.5 (anti), 132.7 (anti), 132.7 (syn), 128.0 (anti), 127.9 (syn), 121.1 (syn), 120.5 (anti), 119.9, 117.75 (syn), 116.9 (anti), 114.5 (anti), 114.2 (syn), 113.8, 113.7, 61.8 (anti), 61.0 (anti), 60.8 (syn), 59.7 (syn), 55.2, 46.9 (anti), 46.6 (syn), 15.4 (anti), 14.2 (anti), 14.1 (syn), 12.4 (syn). MS(DI) m/z 329(M+, 4), 252(6), 228(100), 221(22), 165(27), 146(18), 120(14), 109(16), 91(13), 80(12), 77(15). Found: C, 69.11; H, 6.73; N, 4.09\%. 
Calcd for $\mathrm{C}_{19} \mathrm{H}_{23} \mathrm{NO}_{4}$ : C, 69.28; H, 7.04; N, 4.25\%. HPLC: Daicel Chiralcel OJ-H, Hexane $/ \mathrm{EtOH}=5 / 1$, Flow rate $0.8 \mathrm{~mL} / \mathrm{min}, \mathrm{UV}=244 \mathrm{~nm}, \mathrm{t}_{\mathrm{R}}=22.4 \mathrm{~min}$ (minor, $2 S, 3 S$ ), $\mathrm{t}_{\mathrm{R}}=29.8 \mathrm{~min}$ (major isomer, $2 R, 3 R$ ).<smiles>CCOC(=O)[C@H](C)[C@H](Nc1ccccc1O)c1ccc(F)cc1</smiles>

Ethyl 3-(4-fluorophenyl)-3-N-(2-hydroxyphenyl)amino-2-methylpropionate $(\mathbf{5 c})$ $($ syn/anti $=91 / 9)$

IR $\left(\mathrm{CHCl}_{3}\right)$ 3607, 3420, 2976, 2937, 1724, 1607, 1510, 1448, 1381, 1232, 1157, 1097 , 945, $833 \mathrm{~cm}^{-1}$. $\quad \mathrm{R}_{\mathrm{f}} 0.3$ (Hexane: Ethyl acetate=3:1) ${ }^{1} \mathrm{H}$ NMR $\left(400 \mathrm{MHz}, \mathrm{CDCl}_{3}\right) \delta=$ 7.31-7.19 (m, 2H), 7.00-6.96 (m, 2H), 6.76-6.55 (m, 3H), 6.38 (dd, 1H, J=7.9, $1.3 \mathrm{~Hz}$, syn), 6.31 (dd, $1 \mathrm{H}, J=7.5,1.8 \mathrm{~Hz}$, anti), 5.81 (brs, 1H), 5.26 (brs, 1H), 4.72 (brs, 1H), 4.67 (d, $1 \mathrm{H}, J=5.3 \mathrm{~Hz}$, syn), 4.38 (brs, 1H), 4.33 (d, $1 \mathrm{H}, J=9.0 \mathrm{~Hz}$, anti), 4.18 (q, $2 \mathrm{H}$, $J=7.1 \mathrm{~Hz}$, anti), 4.07 (q, $2 \mathrm{H}, J=7.1 \mathrm{~Hz}, s y n), 2.93$ (dq, $1 \mathrm{H}, J=5.3 \mathrm{~Hz}, 7.1 \mathrm{~Hz}$, syn), 2.85 (dq, $1 \mathrm{H}, J=9.0 \mathrm{~Hz}, 7.0 \mathrm{~Hz}$, anti), 1.26 (t, $3 \mathrm{H}, J=7.1 \mathrm{~Hz}$, anti), $1.21(\mathrm{~d}, 3 \mathrm{H}, J=7.1 \mathrm{~Hz}$, syn), 1.15 (t, $3 \mathrm{H}, J=7.1 \mathrm{~Hz}$, syn), $1.10\left(\mathrm{~d}, 3 \mathrm{H}, J=7.1 \mathrm{~Hz}\right.$, anti). ${ }^{19} \mathrm{~F}$ NMR (376 MHz, $\left.\mathrm{CDCl}_{3}\right) \delta=46.7\left(\mathrm{~s}\right.$, anti), $46.29\left(\mathrm{~s}\right.$, syn). ${ }^{13} \mathrm{C} \mathrm{NMR}\left(75 \mathrm{MHz}, \mathrm{CDCl}_{3}\right) \delta=175.6$ (anti), 174.1 (syn), 163.5, 163.4, 160.2, 145.6 (anti), 143.7 (syn), 136.5, 136.5, 136.4, 135.4 (syn), 134.4 (anti), 128.4 (d, $J_{\mathrm{F}-\mathrm{C}}=7.6 \mathrm{~Hz}$, anti), 128.4 (d, $\left.J_{\mathrm{F}-\mathrm{C}}=8.3 \mathrm{~Hz}, \mathrm{syn}\right), 121.2$ (syn), 120.6 (anti), 119.7 (anti), 117.9 (syn), 116.1, 115.4 (d, $J_{\mathrm{F}-\mathrm{C}}=21.4 \mathrm{~Hz}$, anti), 115.2 (d, $J_{\mathrm{F}-\mathrm{C}}=21.4 \mathrm{~Hz}, \mathrm{syn}$ ), 114.6, 114.2, 113.4, 61.5 (anti), 61.1 (anti), 60.9 (syn), 59.6 (syn), 46.8 (anti), 46.5 (syn), 15.4 (anti), 14.2 (anti), 14.1 (syn), 12.4 (syn). MS(DI) m/z 317( $\left.\mathrm{M}^{+}, 5\right), 217(14), 216(100), 214(14), 213(5), 153(8), 135(6), 120(9), 109(23), 92(6)$, 65(8). Found: C, 67.90; H, 6.35; N, 4.31\%. Calcd for $\mathrm{C}_{18} \mathrm{H}_{20} \mathrm{FNO}_{3}: \mathrm{C}, 68.12 ; \mathrm{H}$, 6.35; N, 4.41\%. HPLC: Daicel Chiralcel OJ-H, Hexane $/ i-\mathrm{PrOH}=9 / 1$, Flow rate 0.7 $\mathrm{mL} / \mathrm{min}, \mathrm{UV}=244 \mathrm{~nm}, \mathrm{t}_{\mathrm{R}}=43.2 \mathrm{~min}$ (minor isomer, $2 S, 3 S$ ), $\mathrm{t}_{\mathrm{R}}=56.2 \mathrm{~min}$ (major isomer, $2 R, 3 R)$. 
<smiles>CCOC(=O)[C@H](C)[C@H](Nc1ccccc1O)c1ccc(Cl)cc1</smiles>

Ethyl 3-(4-chlorophenyl)-3- $N$-(2-hydroxyphenyl)amino-2-methylpropionate $($ syn/anti $=86 / 14)$

IR $\left(\mathrm{CHCl}_{3}\right)$ 3601, 3323, 3028, 3015, 1724, 1611, 1514, 1491, 1448, 1267, 1213, 1205 , 1182, 1092, $1015 \mathrm{~cm}^{-1} . \quad \mathrm{R}_{\mathrm{f}} 0.3$ (Hexane: Ethyl acetate=3:1) ${ }^{1} \mathrm{H}$ NMR (400 MHz, $\left.\mathrm{CDCl}_{3}\right) \delta=7.33-7.17(\mathrm{~m}, 4 \mathrm{H}), 6.78-6.52(\mathrm{~m}, 3 \mathrm{H}), 6.36(\mathrm{dd}, 1 \mathrm{H}, J=7.9,1.5 \mathrm{~Hz}, \mathrm{syn})$, 6.31 (dd, 1H, $J=7.3,1.8 \mathrm{~Hz}$, anti), 5.51 (brs, 1H), 4.76 (brs, 1H), 4.67 (d, $1 \mathrm{H}, J=5.3 \mathrm{~Hz}$, syn), 4.33 (d, $1 \mathrm{H}, J=8.8 \mathrm{~Hz}$, anti), 4.17 (q, $2 \mathrm{H}, J=7.1 \mathrm{~Hz}$, anti), 4.07 (q, $2 \mathrm{H}, J=7.1 \mathrm{~Hz}$, syn), 2.94 (dq, $1 \mathrm{H}, J=5.3 \mathrm{~Hz}, 7.1 \mathrm{~Hz}$, syn), 2.85 (dq, $1 \mathrm{H}, J=8.8 \mathrm{~Hz}, 7.1 \mathrm{~Hz}$, anti), 1.24 (t, $3 \mathrm{H}, J=7.1 \mathrm{~Hz}$, anti), 1.21 (d, $3 \mathrm{H}, J=7.1 \mathrm{~Hz}$, syn), 1.16 (t, $3 \mathrm{H}, J=7.1 \mathrm{~Hz}, s y n), 1.11$ (d, $3 \mathrm{H}, J=7.1 \mathrm{~Hz}$, anti). 13C NMR (75 MHz, $\mathrm{CDCl}_{3}$ ) $\delta=175.4$ (anti), 173.9 (syn), 145.7 (anti), 143.6 (syn), 139.4 (anti), 139.3 (anti), 135.3 (syn), 134.3 (anti), 133.1 (anti), 132.9 (syn), 128.7, 128.5, 128.3, 128.3, 121.3 (syn), 120.7 (anti), 119.9 (anti), 118.0 (syn), 116.3 (anti), 114.6 (anti), 114.3 (syn), 113.6 (syn), 61.6 (anti), 61.1 (anti), 60.9 (syn), 59.7 (syn), 46.6 (anti), 46.3 (syn), 15.4 (anti), 14.3 (anti), 14.2 (syn), 12.3 (syn). MS(DI) m/z 333(M+, 6), 234(27), 233(14), 232(100), 230(12), 169(9), 125(10), 120(12), 115(7), 109(9), 65(10). Found: C, 64.56; H, 5.81; N, 4.19\%. Calcd for $\mathrm{C}_{18} \mathrm{H}_{20} \mathrm{ClNO}_{3}$ : C, 64.77; H, 6.04; N, 4.20\%. HPLC: Daicel Chiralpak AD-H, Hexane/i-PrOH=30/1, Flow rate $0.5 \mathrm{~mL} / \mathrm{min}, \mathrm{UV}=244 \mathrm{~nm}, \mathrm{t}_{\mathrm{R}}=62.4 \mathrm{~min}(2 S, 3 S), \mathrm{t}_{\mathrm{R}}=73.8 \mathrm{~min}$ (major isomer, $2 R, 3 R)$.<smiles>CCOC(=O)[C@H](C)[C@H](Nc1ccccc1O)c1ccc(C)cc1</smiles>

Ethyl 3-N-(2-hydroxyphenyl)amino-2-methyl-3-(4-methylphenyl)propionate 
$($ syn/anti $=94 / 6)$

IR $\left(\mathrm{CHCl}_{3}\right)$ 3601, 3422, 3028, 2984, 2937, 1722, 1611, 1514, 1448, 1373, 1265, 1182, 1042, $816 \mathrm{~cm}^{-1} . \quad \mathrm{R}_{\mathrm{f}} 0.3$ (Hexane: Ethyl acetate=3:1), $1 \mathrm{H}$ NMR (400 MHz, $\mathrm{CDCl}_{3}$ ) $\delta=7.21-7.07(\mathrm{~m}, 4 \mathrm{H}), 6.75-6.52(\mathrm{~m}, 3 \mathrm{H}), 6.43(\mathrm{dd}, 1 \mathrm{H}, J=7.9,1.5 \mathrm{~Hz}, \mathrm{syn}), 6.35$ (dd, $1 \mathrm{H}$, $J=7.5,1.8 \mathrm{~Hz}$, anti), 6.11 (brs, 1H), 5.71 (brs, 1H), 4.69 (d, $1 \mathrm{H}, J=5.5 \mathrm{~Hz}$, syn), 4.29 (d, $1 \mathrm{H}, J=9.3 \mathrm{~Hz}$, anti), 4.18 (q, $2 \mathrm{H}, J=7.1 \mathrm{~Hz}$, anti), 4.07 (q, $2 \mathrm{H}, J=7.1 \mathrm{~Hz}$, syn), 2.95 (dq, $1 \mathrm{H}, J=5.5 \mathrm{~Hz}, 7.0 \mathrm{~Hz}, s y n), 2.87$ (dq, $1 \mathrm{H}, J=9.3 \mathrm{~Hz}, 7.0 \mathrm{~Hz}$, anti), 2.29 (s, $3 \mathrm{H}$, syn), 2.29 (s, $3 \mathrm{H}$, anti), 1.25 (t, $3 \mathrm{H}, J=7.1 \mathrm{~Hz}$, anti), 1.20 (d, $3 \mathrm{H}, J=7.0 \mathrm{~Hz}$, syn), 1.15 (t, 3 $\mathrm{H}, J=7.1 \mathrm{~Hz}, s y n), 1.08\left(\mathrm{~d}, 3 \mathrm{H}, J=7.0 \mathrm{~Hz}\right.$, anti). 13C NMR (100 MHz, $\left.\mathrm{CDCl}_{3}\right)$ $\delta=176.3$ (anti), 174.6 (syn), 146.3 (anti), 144.0 (syn), 137.7 (syn), 137.1 (anti), 136.8, 135.8 (syn), 134.6 (anti), 129.2 (anti), 129.1 (syn), 126.9 (anti), 126.8 (syn), 121.2 (syn), 120.6 (anti), 120.0 (anti), 117.9 (syn), 116.9 (anti), 114.6 (anti), 114.3 (syn), 113.8 (syn), 62.1 (anti), 61.0 (anti), 60.8 (syn), 59.9(syn), 46.8 (anti), 46.4 (syn), 21.0, 15.3 (anti), 14.1 (anti), 14.0 (syn), 12.0 (syn). MS(DI) m/z 313( $\left.\mathrm{M}^{+}, 5\right), 213(15), 212(100)$, 210(15), 149(11), 120(12), 109(5), 105(13), 93(5), 91(9), 65(10). Found: C, 72.75; H, 7.76; N, 4.35\%. Calcd for $\mathrm{C}_{19} \mathrm{H}_{23} \mathrm{NO}_{3}$ : C, 72.82; H, 7.40; N, 4.47\%. HPLC: Daicel Chiralpak AD-H, Hexane/EtOH=40/1, Flow rate $0.5 \mathrm{~mL} / \mathrm{min}, \mathrm{UV}=244 \mathrm{~nm}, \mathrm{t}_{\mathrm{R}}=46.4$ $\min \left(\right.$ minor isomer, $2 S, 3 S$ ), $\mathrm{t}_{\mathrm{R}}=56.2 \mathrm{~min}$ (major isomer, $2 R, 3 R$ ).

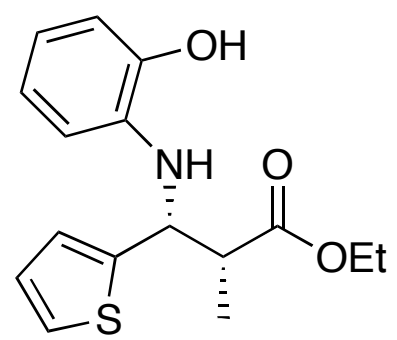

Ethyl 3- $N$-(2-hydroxyphenyl)amino-2-methyl-3-(2-thienyl)propionate $($ syn/anti $=94 / 6)$

IR $\left(\mathrm{CHCl}_{3}\right)$ 3601, 3412, 3028, 2986, 1724, 1611, 1514, 1448, 1373, 1267, 1231, 1202 , $1167 \mathrm{~cm}^{-1}$. $\quad \mathrm{R}_{\mathrm{f}} 0.3$ (Hexane: Ethyl acetate=3:1) ${ }^{1} \mathrm{H}$ NMR $\left(400 \mathrm{MHz}, \mathrm{CDCl}_{3}\right)$ $\mathrm{d}=7.20-7.16(\mathrm{~m}, 1 \mathrm{H}), 6.96-6.90(\mathrm{~m}, 2 \mathrm{H}), 6.77-6.51(\mathrm{~m}, 4 \mathrm{H}), 5.01(\mathrm{~d}, 1 \mathrm{H}, J=5.7 \mathrm{~Hz}$, syn), 4.83 (brs, 1H), 4.72 (d, $1 \mathrm{H}, J=8.4 \mathrm{~Hz}$, anti), 4.50 (brs, 1H), 4.19 (q, $2 \mathrm{H}, J=7.1 \mathrm{~Hz}$, anti), 4.09 (q, $2 \mathrm{H}, J=7.1 \mathrm{~Hz}$, syn), 3.04 (dq, $1 \mathrm{H}, J=7.2,5.7 \mathrm{~Hz}$, syn), 2.96 (dq, $1 \mathrm{H}$, $J=8.4,7.0 \mathrm{~Hz}$, anti), 1.31 (d, $3 \mathrm{H}, J=7.2 \mathrm{~Hz}$, syn), 1.26 (t, $3 \mathrm{H}, J=7.1 \mathrm{~Hz}$, anti), 1.19 (t, 3 
$\mathrm{H}, J=7.1 \mathrm{~Hz}$, syn), $1.19\left(\mathrm{~d}, 3 \mathrm{H}, J=7.0 \mathrm{~Hz}\right.$, anti). ${ }^{13} \mathrm{C} \mathrm{NMR}\left(75 \mathrm{MHz}, \mathrm{CDCl}_{3}\right) \delta=175.7$ (anti), 173.9 (syn), 147.2 (anti), 145.4 (syn), 144.9 (anti), 144.5 (syn), 135.1 (syn), 133.7 (anti), 126.6 (anti), 126.6 (syn), 125.5 (anti), 124.6 (syn), 124.4 (anti), 124.2 (syn), 121.5 (anti), 121.2 (syn), 120.4 (anti), 119.0 (syn), 118.4 (anti), 114.9 (anti), 114.7 (syn), 114.6 (syn), 61.2 (anti), 61.0 (syn), 58.7 (anti), 56.8 (syn), 47.3 (anti), 46.6 (syn), 15.4 (anti), 14.3 (anti), 14.2 (syn), 12.8 (syn). MS(DI) m/z 305(M+, 4), 228(7), 204(40), 197(10), 123(10), 121(4), 120(7), 109(13), 97(15), 87(11), 83(100), 79(19), 74(11), 65(9). Found: C, 62.55; H, 6.49; N, 4.25; S, 10.39\%. Calcd for $\mathrm{C}_{16} \mathrm{H}_{19} \mathrm{NO}_{3} \mathrm{~S}$ : Calcd C, 62.93; H, 6.27; N, 4.59; S, 10.50\%. HPLC: Daicel Chiralcel OD-H, Hexane $/ i-\mathrm{PrOH}=40 / 1$, Flow rate $0.5 \mathrm{~mL} / \mathrm{min}, \mathrm{UV}=244 \mathrm{~nm}, \mathrm{t}_{\mathrm{R}}=69.7 \mathrm{~min}$ (minor isomer, $2 S, 3 S$ ), $\mathrm{t}_{\mathrm{R}}=80.7 \mathrm{~min}$ (major isomer, $2 R, 3 R$ ).<smiles>CCOC(=O)[C@H](C)[C@H](/C=C/c1ccccc1)Nc1ccccc1O</smiles>

Ethyl (E)-3-N-(2-hydroxyphenyl)amino-2-methyl-5-phenylpent-4-enoate (5g) (syn/anti $=95 / 5)$

IR $\left(\mathrm{CHCl}_{3}\right)$ 3601, 3354, 3015, 2984, 1724, 1611, 1514, 1448, 1263, 1221, 1190, 966 $\mathrm{cm}^{-1} . \quad \mathrm{R}_{\mathrm{f}} 0.3$ (Hexane: Ethyl acetate=3:1), ${ }^{1} \mathrm{H} \mathrm{NMR}\left(400 \mathrm{MHz}, \mathrm{CDCl}_{3}\right) \delta=7.44-7.19$ (m, 5H), 6.80-6.63 (m, 4H), 6.55 (d, $1 \mathrm{H}, J=15.9 \mathrm{~Hz}$, syn), 6.44 (d, $1 \mathrm{H}, J=15.7 \mathrm{~Hz}$, anti), 6.15 (dd, $1 \mathrm{H}, J=6.6 \mathrm{~Hz}, 15.9 \mathrm{~Hz}$, syn), 6.01 (dd, $1 \mathrm{H}, J=15.7,7.4 \mathrm{~Hz}$, anti), 4.20 (dd, 1 $\mathrm{H}, J=6.6,5.1 \mathrm{~Hz}, s y n), 4.15$ (q, 2 H, J=7.1 Hz, syn), 4.12 (q, $2 \mathrm{H}, J=7.1 \mathrm{~Hz}$, anti), 4.03 (dd, $1 \mathrm{H}, J=7.4 \mathrm{~Hz}, 8.1 \mathrm{~Hz}$, anti), 2.88 (dq, $1 \mathrm{H}, J=7.1,5.1 \mathrm{~Hz}$, syn), 2.76 (dq, $1 \mathrm{H}$, $J=8.1,7.1 \mathrm{~Hz}$, anti), 1.30 (t, $3 \mathrm{H}, J=7.1 \mathrm{~Hz}$, syn), 1.27 (d, $3 \mathrm{H}, J=7.1 \mathrm{~Hz}$, anti), 1.23 (t, 3 $\mathrm{H}, J=7.1 \mathrm{~Hz}, s y n), 1.23\left(\mathrm{~d}, 3 \mathrm{H}, J=7.1 \mathrm{~Hz}\right.$, anti). ${ }^{13} \mathrm{C} \mathrm{NMR}\left(100 \mathrm{MHz}, \mathrm{CDCl}_{3}\right)$ $\delta=175.6$ (anti), 174.3 (syn), 146.4 (anti), 144.9 (syn), 136.5 (syn), 136.4 (anti), 135.2 (syn), 134.3 (anti), 132.6 (anti), 132.0 (syn), 128.4, 128.2, 127.5, 127.5, 126.4, 126.3, 121.1 (syn), 120.7 (anti), 120.3 (anti), 119.0 (syn), 117.5 (anti), 115.3 (anti), 114.8 (syn), 114.6 (syn), 61.0 (anti), 60.9 (syn), 60.2 (anti), 59.1 (syn), 45.1 (anti), 44.5 (syn), 14.6 (anti), 14.3, 13.1 (syn). MS(DI) m/z 325(M+, 4), 225(17), 224(100), 222(12), 220(14), 216(16), 171(11), 143(83), 142(19), 141(16), 131(7), 129(18), 128(32), 116(7), 115(31), 
109(24), 103(6), 102(6), 91(33), 80(12), 77(20), 74(14), 65(20). Found: C, 73.43; H, 6.97; N, 4.15\%. Calcd for $\mathrm{C}_{20} \mathrm{H}_{23} \mathrm{NO}_{3}: \mathrm{C}, 73.82 ; \mathrm{H}, 7.12 ; \mathrm{N}, 4.30 \%$. HPLC: Daicel Chiralcel OD-H, Hexane $/ i-\mathrm{PrOH}=15 / 1$, Flow rate $0.5 \mathrm{~mL} / \mathrm{min}, \mathrm{UV}=244 \mathrm{~nm}, \mathrm{t}_{\mathrm{R}}=29.3$ $\min \left(\right.$ minor isomer, $2 S, 3 R$ ), $\mathrm{t}_{\mathrm{R}}=33.5 \mathrm{~min}$ (major isomer, $2 R, 3 S$ ).

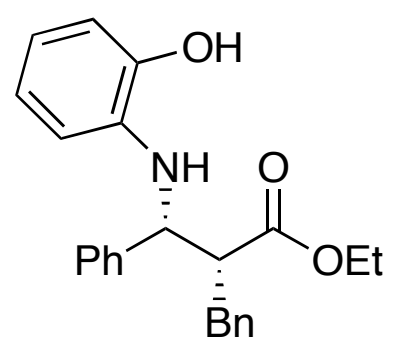

Ethyl 2-benzyl-3- $N$-(2-hydroxyphenyl)amino-3-phenylpropionate (5h) (syn/anti=93/7) IR $\left(\mathrm{CHCl}_{3}\right)$ 3601, 3420, 3026, 3009, 1720, 1611, 1495, 1454, 1377, 1267, 1219, 1205, $1167 \mathrm{~cm}^{-1}$. $\quad \mathrm{R}_{\mathrm{f}} 0.3$ (Hexane : Ethyl acetate=3:1) ${ }^{1} \mathrm{H}$ NMR $\left(400 \mathrm{MHz}, \mathrm{CDCl}_{3}\right) \delta=$ 7.38-7.10 (m, 10H), 6.75-6.54 (m, 3H), 6.43 (d, 1H, J=7.1 Hz, syn), 6.30-6028 (m, 1H, anti), 5.59 (brs, 1H, anti), 5.24 (brs, 1H, syn), 4.90 (brs, 1H, syn, anti), 4.70 (d, $1 \mathrm{H}$, $J=5.9 \mathrm{~Hz}$, syn), 4.44 (d, 1H, J=7.1 Hz, anti), 3.99 (q, $2 \mathrm{H}, J=7.1 \mathrm{~Hz}$, anti), 3.90 (q, $2 \mathrm{H}$, $J=7.1 \mathrm{~Hz}$, syn), 3.20-3.08 (m, 2H), 3.00-2.79 (m, 1H), 1.00 (t, 3 H, J=7.1 Hz, anti), 0.96 $(\mathrm{t}, 3 \mathrm{H}, J=7.1 \mathrm{~Hz}, \mathrm{syn}) ;{ }^{13} \mathrm{C} \mathrm{NMR}\left(100 \mathrm{MHz}, \mathrm{CDCl}_{3}\right) \delta=174.6$ (anti), 173.1 (syn), 145.1 (anti), 143.7 (syn), 141.0 (anti), 140.6 (syn), 139.2 (syn), 138.5 (anti), 135.8 (syn), 134.9 (anti), 128.8, 128.8, 128.6, 128.5, 128.4, 128.4, 127.5, 127.0, 126.8, 126.5, 126.3, 121.4 (syn), 121.0 (anti), 118.9 (anti), 117.9 (syn), 115.2 (anti), 114.6 (anti), 114.4 (syn), 113.5 (syn), 60.8 (anti), 60.7 (syn), 59.9, 54.9 (anti), 54.8 (syn), 36.4 (anti), 33.9 (syn), 13.9 (anti), 13.8 (syn); MS(DI) m/z 375(M+2, 2), 200(2), 198(100), 193(6), 178(3), 152(2), 131(4), 120(1), 115(5), 109(4), 104(4), 93(4), 91(32), 80(5), 78(3), 77(7), 65(11); Found: C, 76.54; H, 6.64; N, 3.79\%. Calcd for $\mathrm{C}_{24} \mathrm{H}_{25} \mathrm{NO}_{3}$ : C, 76.77; $\mathrm{H}, 6.71$; N, 3.73\%. HPLC: Daicel Chiralpak AS-H, Hexane/i-PrOH=30/1, Flow rate 0.5 $\mathrm{mL} / \mathrm{min}, \mathrm{UV}=244 \mathrm{~nm}, \mathrm{t}_{\mathrm{R}}=54.9 \mathrm{~min}$ (minor isomer, $2 S, 3 S$ ), $\mathrm{t}_{\mathrm{R}}=64.4$ min (major isomer, $2 R, 3 R)$. 
<smiles>CCOC(=O)[C@H](Cc1ccccc1)[C@H](Nc1ccccc1O)c1ccc(OC)cc1</smiles>

Ethyl 2-benzyl-3- $N$-(2-hydroxyphenyl)amino-3-(4-methoxylphenyl)propionate $($ syn/anti $=93 / 7)$

IR $\left(\mathrm{CHCl}_{3}\right) 3601,3418,3009,2980,1720,1611,1512,1448,1377,1248,1177,1109$, 1036, $831 \mathrm{~cm}^{-1}$. $\quad \mathrm{R}_{\mathrm{f}} 0.3$ (Hexane: Ethyl acetate=3:1) ${ }^{1} \mathrm{H}$ NMR (400 $\mathrm{MHz}, \mathrm{CDCl}_{3}$ ) $\delta=7.29-7.09(\mathrm{~m}, 2 \mathrm{H}), 6.91-6.81(\mathrm{~m}, 2 \mathrm{H}), 6.75-6.54(\mathrm{~m}, 3 \mathrm{H}), 6.43(\mathrm{~d}, 1 \mathrm{H}, J=7.7 \mathrm{~Hz}$, syn), 6.32-6.29 (m, 1H, anti), 5.66 (brs, $1 \mathrm{H}$, anti), 5.32 (brs, $1 \mathrm{H}$, syn), 4.84 (brs, $1 \mathrm{H}$, syn), 4.78 (brs, $1 \mathrm{H}$, anti), 4.65 (d, $1 \mathrm{H}, J=5.9 \mathrm{~Hz}$, syn), 4.39 (d, $1 \mathrm{H}, J=7.5 \mathrm{~Hz}$, anti), 4.01 (q, $2 \mathrm{H}, J=7.1 \mathrm{~Hz}$, anti), 3.91 (q, $2 \mathrm{H}, J=7.1 \mathrm{~Hz}$, syn), 3.77 (s, $3 \mathrm{H}$, syn), 3.76 (s, 3 $\mathrm{H}$, anti), 3.18-2.90 (m, 3H), 2.78 (dd, 1H, $J=13.3,5.9 \mathrm{~Hz}$, anti), 1.03 (t, $3 \mathrm{H}, J=7.1 \mathrm{~Hz}$, anti), 0.99 (t, $3 \mathrm{H}, J=7.1 \mathrm{~Hz}$, syn). ${ }^{13} \mathrm{C} \mathrm{NMR}\left(75 \mathrm{MHz}, \mathrm{CDCl}_{3}\right) \delta=174.6$ (anti), 173.0 (syn), 158.7, 145.2 (anti), 143.7 (syn), 139.1 (syn), 138.5 (anti), 135.6 (syn), 134.7 (anti), 132.8 (anti), 132.4 (syn), 128.7, 128.2, 128.0, 127.8, 126.3 (anti), 126.2 (syn), 121.2 (syn), 120.8 (anti), 118.9 (anti), 117.7 (syn), 115.4 (anti), 114.4 (anti), 114.2 (syn), 113.9 (anti), 113.8 (syn), 113.4 (syn), 60.8 (anti), 60.7 (syn), 59.6 (anti), 59.4 (syn), 55.2, 55.1 (anti), 54.9 (syn), 36.5 (anti), 34.2 (syn), 14.1 (anti), 14.0 (syn). MS(DI) m/z 405( $\left.\mathrm{M}^{+}, 2\right), 296(2), 229(16), 228(100), 226(10), 222(5), 189(20), 145(5), 121(8)$, 120(12), 116(8), 115(6), 109(6), 91(41), 77(5), 65(7). Found: C, 74.22; H, 6.87; N, 3.25\%. Calcd for $\mathrm{C}_{25} \mathrm{H}_{27} \mathrm{NO}_{4}$ : C, 74.05; H, 6.71; N, 3.45\%. HPLC: Daicel Chiralpak AD-H, Hexane $/ i-\mathrm{PrOH}=5 / 1$, Flow rate $=0.5 \mathrm{~mL} / \mathrm{min}, \mathrm{UV}=244 \mathrm{~nm}, \mathrm{t}_{\mathrm{R}}=21.7 \mathrm{~min}($ minor isomer, $2 S, 3 S$ ), $\mathrm{t}_{\mathrm{R}}=34.7 \mathrm{~min}$ (major isomer, $2 R, 3 R$ ). 


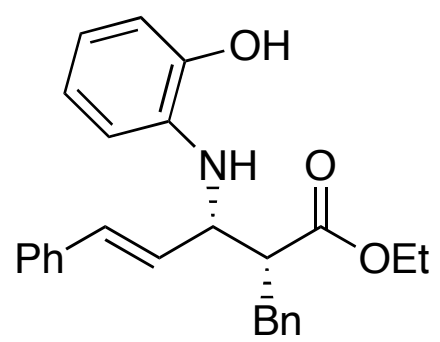

Ethyl (E)-3-N-(2-hydroxyphenyl)amino-2-methyl-5-phenylpent-4-enoate (syn/anti=95/5)

IR $\left(\mathrm{CHCl}_{3}\right)$ 3609, 3337, 3011, 2980, 2930, 1719, 1611, 1516, 1497, 1448, 1377, 1232, 1165, 1040, 966, $945 \mathrm{~cm}^{-1}$. $\quad \mathrm{R}_{\mathrm{f}} 0.4$ (Hexane: Ethyl acetate=3:1), ${ }^{1} \mathrm{H}$ NMR (400 MHz, $\left.\mathrm{CDCl}_{3}\right) \delta=7.36-7.15(\mathrm{~m}, 10 \mathrm{H}), 6.79-6.62(\mathrm{~m}, 4 \mathrm{H}), 6.59(\mathrm{~d}, 1 \mathrm{H}, J=15.7 \mathrm{~Hz}$, syn), 6.46 (d, $1 \mathrm{H}, J=16.0 \mathrm{~Hz}$, anti), 6.19 (dd, $1 \mathrm{H}, J=15.7,7.1 \mathrm{~Hz}$, syn), 6.08 (d, $1 \mathrm{H}, J=16.0,7.1$ $\mathrm{Hz}$, anti), 5.50 (brs, $1 \mathrm{H}$ ), 5.21 (brs, $1 \mathrm{H}$ ), 4.42 (brs, $1 \mathrm{H}), 4.19$ (brs, $1 \mathrm{H}), 4.15-4.02$ (m, $3 \mathrm{H}), 3.17-2.96(\mathrm{~m}, 3 \mathrm{H}), 1.14(\mathrm{t}, 3 \mathrm{H}, J=7.1 \mathrm{~Hz}, s y n), 1.10$ (t, $3 \mathrm{H}, J=7.1 \mathrm{~Hz}$, anti). ${ }^{13} \mathrm{C}$ NMR $\left(75 \mathrm{MHz}, \mathrm{CDCl}_{3}\right) \delta=173.1,144.3,138.8,136.4,135.2,132.4,128.8,128.4$, $128.3,127.7,127.5,126.4,126.3,121.2,118.5,114.5,114.2,60.8,57.7,52.3,34.7$, 14.2. MS(DI) m/z 401(M+, 2), 292(14), 225(17), 224(100), 222(17), 220(17), 219(16), 218(13), 217(11), 202(9), 189(13), 131(9), 129(16), 120(9), 117(15), 115(33), 109(29), 105(10), 104(13), 92(13), 91(92), 80(12), 76(16), 64(20). Found: C, 77.66; H, 6.56; N, 3.19\%. Calcd for $\mathrm{C}_{26} \mathrm{H}_{27} \mathrm{NO}_{3}$ : C, 77.78; H, 6.78; N, 3.49\%. HPLC: Daicel Chiralcel OD-H, Hexane $/ i-\mathrm{PrOH}=30 / 1$, Flow rate $1.0 \mathrm{~mL} / \mathrm{min}, \mathrm{UV}=244 \mathrm{~nm}, \mathrm{t}_{\mathrm{R}}=30.6 \mathrm{~min}(2 S$, $3 R), \mathrm{t}_{\mathrm{R}}=58.2 \min (2 R, 3 S)$.

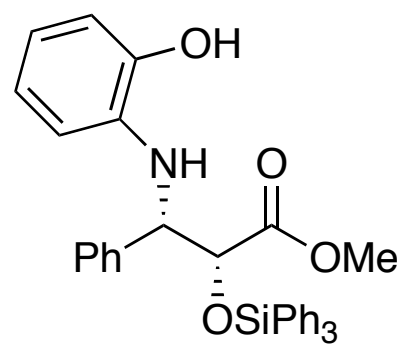

Methyl 3- $N$-(2-hydroxyphenyl)amino-3-phenyl-2-triphenylsiloxypropionate $($ syn/anti $=100 / 0)$

$[\alpha]_{\mathrm{D}}^{25}+27.8\left(\mathrm{c} 1.03, \mathrm{CHCl}_{3}\right)\left(91 \%\right.$ ee). amorphous solid. IR $\left(\mathrm{CHCl}_{3}\right) 3603,3329$, 3009, 2978, 1753, 1611, 1514, 1454, 1429, 1273, 1119, $1086 \mathrm{~cm}^{-1} ; \quad \mathrm{R}_{\mathrm{f}}$ 0.3(Hexane: Ethyl acetate=3:1) $\quad{ }^{1} \mathrm{H}$ NMR $\left(400 \mathrm{MHz}, \mathrm{CDCl}_{3}\right) \delta=7.40-7.23(\mathrm{~m}, 20 \mathrm{H}), 6.69(\mathrm{~d}, 1 \mathrm{H}$, 
$J=7.5 \mathrm{~Hz}), 6.62(\mathrm{dd}, 1 \mathrm{H}, J=7.5,7.5 \mathrm{~Hz}), 6.51(\mathrm{dd}, 1 \mathrm{H}, J=7.5,7.5 \mathrm{~Hz}), 6.35(\mathrm{~d}, 1 \mathrm{H}$, $J=7.5 \mathrm{~Hz}$ ), 5.74 (brs, 1H), 5.36 (d, $1 \mathrm{H}, J=8.3 \mathrm{~Hz}), 4.92$ (d, $1 \mathrm{H}, J=8.3 \mathrm{~Hz}$ ), 4.70 (brs, 1 $\mathrm{H}), 3.35(\mathrm{~s}, 3 \mathrm{H}) ;{ }^{13} \mathrm{C}$ NMR $\left(100 \mathrm{MHz}, \mathrm{CDCl}_{3}\right) \delta=172.0,144.0,139.3,135.5,135.4$, $133.1,130.0,128.5,127.7,127.4,127.4,121.1,117.7,114.6,113.4,77.4,60.5,51.9$; MS(DI) m/z 545(M+1 1), 510(1), 482(3), 456(3), 433(1), 278(5), 359(2), 276(7), 271(8), 260(4), 259(15), 213(6), 198(100), 181(4), 167(2), 152(1), 122(2), 120(10), 105(2), 91(3), 84(1), 77(3), 65(1); Found: C,74.54; H, 5.61; N, 2.37\%. Calcd for $\mathrm{C}_{34} \mathrm{H}_{31} \mathrm{NO}_{4} \mathrm{Si}: \mathrm{C}, 74.83 ; \mathrm{H}$, 5.73; N, 2.57\%. HPLC: Daicel Chiralpak AS-H, Hexane $/ \mathrm{EtOH}=50 / 1$, Flow rate $0.5 \mathrm{~mL} / \mathrm{min}, \mathrm{UV}=244 \mathrm{~nm}, \mathrm{t}_{\mathrm{R}}=37.8 \mathrm{~min}$ (minor isomer, $2 S, 3 R$ ), $\mathrm{t}_{\mathrm{R}}=42.9 \mathrm{~min}$ (major isomer, $2 R, 3 S$ ).

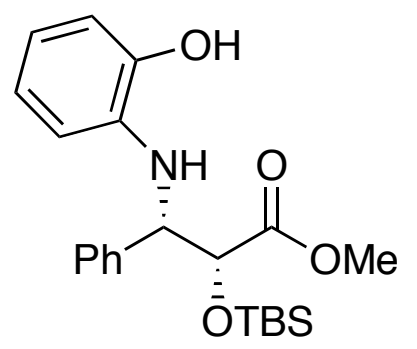

Methyl 2-tert-butyldimethylsiloxy-3- $N$-(2-hydroxyphenyl)amino-3-phenylpropionate (5l) $($ syn/anti=94/6)

IR $\left(\mathrm{CHCl}_{3}\right)$ 3676, 3603, 3422, 3017, 2955, 2932, 2858, 1747, 1611, 1514, 1454, 1362, $1259,1227,1204,1128,839 \mathrm{~cm}^{-1} ; \quad \mathrm{R}_{\mathrm{f}} 0.4$ (Hexane: Ethyl acetate=3:1) ${ }^{1} \mathrm{H}$ NMR $(400$ $\left.\mathrm{MHz}, \mathrm{CDCl}_{3}\right) \delta=7.34-7.19(\mathrm{~m}, 5 \mathrm{H}), 6.75-6.36$ (m, 4H), 5.73 (brs, 1H), 5.19 (brs, $1 \mathrm{H}$ ), 4.91 (d, 1H, $J=1.8 \mathrm{~Hz}$, syn), 4.70 (d, $1 \mathrm{H}, J=5.5 \mathrm{~Hz}$, anti), 4.56 (d, $1 \mathrm{H}, J=5.5 \mathrm{~Hz}$, anti), $4.48(\mathrm{~d}, 1 \mathrm{H}, J=1.8 \mathrm{~Hz}, \mathrm{syn}), 3.72$ (s, $3 \mathrm{H}$, syn), 3.64 (s, $3 \mathrm{H}$, anti), 0.87 (s, $9 \mathrm{H}$, anti), 0.81 (s, $9 \mathrm{H}$, syn),-0.05 (s, $3 \mathrm{H}$, anti),-0.08 (s, 3H, anti), -0.13 (s, $3 \mathrm{H}$, syn),-0.38 (s, 3 $\mathrm{H}$, syn); ${ }^{13} \mathrm{C}$ NMR (100 MHz, $\mathrm{CDCl}_{3}$ ) $\delta=172.8$ (syn), 172.3 (anti), 144.3, 139.7 (syn), 138.8 (anti), 135.4 (syn), 135.1 (anti), 128.3, 128.1, 127.8, 127.7, 127.4, 127.2, 121.3 (anti), 121.1 (syn), 118.3 (anti), 117.9 (syn), 114.7 (syn), 114.4 (anti), 114.1 (anti), 113.9 (syn), 76.7 (syn), 75.7 (anti), 60.9 (anti), 60.6 (syn), 52.3 (syn), 51.8 (anti), 25.6 (anti), 25.6 (syn), 18.2 (syn), 14.2 (anti), -5.3 (anti), -5.6 (syn), -5.6 (anti), -6.1 (syn); MS(DI) m/z 402(M+, 1), 309(3), 279(3), 253(3), 210(2), 198(100), 167(4), 161(2), 152(2), 146(3), 131(4), 120(19), 115(2), 104(6), 93(6), 91(12), 89(14), 77(14), 75(35), 73(19), 65(11); Found: C, 65.96; H, 7.49; N, 3.34\%. Calcd for $\mathrm{C}_{22} \mathrm{H}_{31} \mathrm{NO}_{4} \mathrm{Si}: \mathrm{C}$, 
65.80; H, 7.78; N, 3.49\%. HPLC: Daicel Chiralpak AD-H, Hexane/EtOH=25/1, Flow rate $0.5 \mathrm{~mL} / \mathrm{min}, \mathrm{UV}=244 \mathrm{~nm}, \mathrm{t}_{\mathrm{R}}=11.1 \mathrm{~min}(2 S, 3 R), \mathrm{t}_{\mathrm{R}}=17.4 \mathrm{~min}(2 R, 3 S)$.<smiles>COC(=O)C(C)(C)C(N)c1ccccc1</smiles>

Methyl 3-amino-3-phenyl-2,2-dimethylpropionate

To

a suspension

of

methyl

3-(N-2-hydroxyphenylamino)-2,2-dimethyl-3-phenylpropionate (4a) (99 $\mathrm{mg}, 0.33$ $\mathrm{mmol})$ and $\mathrm{K}_{2} \mathrm{CO}_{3}(99 \mathrm{mg}, 0.33 \mathrm{mmol})$ in acetone $(5 \mathrm{~mL})$ was added methyl iodide (1 $\mathrm{mL}$ ) and the mixture was stirred at room temperature for $10 \mathrm{~h}$. The reaction mixture was quenched by addition of water at $0{ }^{\circ} \mathrm{C}$ and extracted with ethyl acetate. The combined organic layers were washed with brine, dried over anhydrous $\mathrm{Na}_{2} \mathrm{SO}_{4}$. After filtration of the drying agent, the filtrate was concentrated to dryness in vacuo. Purification by $p$-TLC gave a methyl ether $(82 \mathrm{mg}, 0.26 \mathrm{mmol})$ in a quantitative yield. To a solution of the methyl ether $(105 \mathrm{mg}, 0.33 \mathrm{mmol})$ in $\mathrm{CH}_{3} \mathrm{CN}(3 \mathrm{~mL})$ was added a solution of CAN (426 mg, $0.78 \mathrm{mmol})$ in water $(3 \mathrm{~mL})$ at $0{ }^{\circ} \mathrm{C}$ for $2 \mathrm{~min}$. After being stirred at the temperature for $1 \mathrm{~h}$, another portion of CAN (277 mg, $0.51 \mathrm{mmol})$ in $\mathrm{H}_{2} \mathrm{O}$ $(1.5 \mathrm{~mL})$ was added and the stirring was continued at room temperature for $2.5 \mathrm{~h}$. $\mathrm{K}_{2} \mathrm{CO}_{3}$ and $\mathrm{Na}_{2} \mathrm{SO}_{3}$ was added and the $\mathrm{pH}$ of the solution was adjusted to 8-9. After filtration over Celite, the filtrate was extracted with $\mathrm{CH}_{2} \mathrm{Cl}_{2}$ for 3 times. The combined organic layers was successively washed with aq. $\mathrm{Na}_{2} \mathrm{SO}_{3}$, brine, and dried over anhydrous $\mathrm{Na}_{2} \mathrm{SO}_{4}$ and concentrated to dryness in vacuo. The crude mixture was purified by $\mathrm{SiO}_{2}$ column chromatography to give the title compounds in $47 \%$ yield.

$[\alpha]_{\mathrm{D}}^{21}+33.3$ (c 1.05, 1N HCl); lit $^{8}[\alpha]_{\mathrm{D}^{20}}+34.2$ (c $\left.0.331 \mathrm{~N} \mathrm{HCl}\right) . \mathrm{R}_{\mathrm{f}} 0.2$ (Hexane: Ethyl acetate $=1: 1),{ }^{1} \mathrm{H}$ NMR $\left(400 \mathrm{MHz}, \mathrm{CDCl}_{3}\right) \delta=7.33-7.24(\mathrm{~m}, 5 \mathrm{H}), 4.23(\mathrm{~s}, 1 \mathrm{H}), 3.69$ (s, $3 \mathrm{H}), 1.69$ (brs, $2 \mathrm{H}), 1.15(\mathrm{~s}, 3 \mathrm{H}), 1.08(\mathrm{~s}, 3 \mathrm{H}) .{ }^{13} \mathrm{C} \mathrm{NMR}\left(100 \mathrm{MHz}, \mathrm{CDCl}_{3}\right) \delta=$ $177.8,141.9,128.1,127.8,127.3,61.9,51.8,47.8,23.6,19.4$.

\section{Cartesian coordinates and absolute electronic energies of stationary points}

The Cartesian coordinates of all stationary points are listed. For each structure, absolute electronic energies obtained at the BHandHLYP/6-31G* level are shown in parentheses. For the located transition state structures, the number of negative eigenvalues of the Hessian at the converged structures are also indicated $($ nimag $=1)$. 


\section{CP1i}

$(\mathrm{E}(\mathrm{RB}+\mathrm{HF}-\mathrm{LYP})=-1736.19724160 \quad$ A.U. $)$

\begin{tabular}{|c|c|c|c|c|c|}
\hline \multirow{3}{*}{$\begin{array}{l}\text { Center } \\
\text { Number }\end{array}$} & Atomic & \multirow{2}{*}{$\begin{array}{c}\text { Atomic } \\
\text { Type }\end{array}$} & \multicolumn{3}{|c|}{ Coordinates (Angstroms) } \\
\hline & Number & & $\mathrm{X}$ & $\mathrm{Y}$ & Z \\
\hline & ------- & ------- & ---------- & ---------- & ---------- \\
\hline 1 & 6 & 0 & -2.137820 & 2.504741 & 1.305602 \\
\hline 2 & 1 & 0 & -2.615098 & 4.585173 & 1.225819 \\
\hline 3 & 6 & 0 & -2.887521 & 3.594626 & 0.898884 \\
\hline 4 & 6 & 0 & -3.595205 & 1.016257 & 0.066038 \\
\hline 5 & 6 & 0 & -3.987779 & 3.407038 & 0.075708 \\
\hline 6 & 6 & 0 & -2.491926 & 1.232030 & 0.892618 \\
\hline 7 & 6 & 0 & -4.331116 & 2.130615 & -0.333247 \\
\hline 8 & 1 & 0 & -5.175490 & 1.986497 & -0.987465 \\
\hline 9 & 1 & 0 & -4.573302 & 4.250939 & -0.250646 \\
\hline 10 & 6 & 0 & -3.030885 & -1.275915 & -0.767097 \\
\hline 11 & 6 & 0 & -3.983625 & -0.345835 & -0.350486 \\
\hline 12 & 6 & 0 & -4.725286 & -2.920522 & -1.159412 \\
\hline 13 & 6 & 0 & -5.319434 & -0.743719 & -0.351542 \\
\hline 14 & 6 & 0 & -3.392134 & -2.550556 & -1.164918 \\
\hline 15 & 6 & 0 & -5.692711 & -2.013778 & -0.753044 \\
\hline 16 & 1 & 0 & -6.732218 & -2.297640 & -0.738282 \\
\hline 17 & 1 & 0 & -6.068506 & -0.046836 & -0.012372 \\
\hline 18 & 1 & 0 & -5.006079 & -3.914378 & -1.467705 \\
\hline 19 & 8 & 0 & -1.715884 & -0.905949 & -0.873890 \\
\hline 20 & 8 & 0 & -1.784910 & 0.166852 & 1.380681 \\
\hline 21 & 15 & 0 & -0.781904 & -0.683468 & 0.431105 \\
\hline 22 & 8 & 0 & -0.481554 & -1.959989 & 1.127985 \\
\hline 23 & 8 & 0 & 0.351750 & 0.182352 & -0.029474 \\
\hline 24 & 1 & 0 & 1.884401 & -0.120369 & 0.055352 \\
\hline 25 & 1 & 0 & 4.171069 & -5.417964 & 0.009318 \\
\hline 26 & 6 & 0 & 3.927133 & -4.368302 & -0.008993 \\
\hline 27 & 6 & 0 & 3.289307 & -1.690326 & -0.050400 \\
\hline 28 & 6 & 0 & 3.070157 & -3.850602 & 0.945641 \\
\hline 29 & 6 & 0 & 4.471025 & -3.550069 & -0.990482 \\
\hline 30 & 6 & 0 & 4.145185 & -2.207159 & -1.013941 \\
\hline 31 & 6 & 0 & 2.735032 & -2.501110 & 0.943740 \\
\hline 32 & 1 & 0 & 2.650832 & -4.472691 & 1.717988 \\
\hline 33 & 1 & 0 & 5.125740 & -3.957869 & -1.741995 \\
\hline 34 & 1 & 0 & 4.517911 & -1.568215 & -1.798465 \\
\hline 35 & 7 & 0 & 2.921415 & -0.317421 & -0.061501 \\
\hline 36 & 6 & 0 & 3.763551 & 0.629819 & -0.250771 \\
\hline 37 & 1 & 0 & 4.799162 & 0.330958 & -0.327499 \\
\hline 38 & 6 & 0 & 3.486753 & 2.043066 & -0.362541 \\
\hline 39 & 6 & 0 & 3.140168 & 4.785042 & -0.577249 \\
\hline 40 & 6 & 0 & 4.595006 & 2.890627 & -0.463620 \\
\hline 41 & 6 & 0 & 2.194085 & 2.581894 & -0.376139 \\
\hline 42 & 6 & 0 & 2.034282 & 3.949703 & -0.483422 \\
\hline 43 & 6 & 0 & 4.423267 & 4.256573 & -0.567676 \\
\hline 44 & 1 & 0 & 5.589590 & 2.472967 & -0.456619 \\
\hline 45 & 1 & 0 & 1.331416 & 1.938687 & -0.309544 \\
\hline 46 & 1 & 0 & 1.040292 & 4.364541 & -0.496051 \\
\hline 47 & 1 & 0 & 5.279961 & 4.905022 & -0.642390 \\
\hline 48 & 1 & 0 & 3.001386 & 5.850695 & -0.660904 \\
\hline 49 & 8 & 0 & 1.975870 & -1.955708 & 1.901386 \\
\hline 50 & 1 & 0 & 1.001630 & -2.045105 & 1.695112 \\
\hline 51 & 1 & 0 & -2.617748 & -3.229785 & -1.477131 \\
\hline 52 & 1 & 0 & -1.285511 & 2.614045 & 1.954412 \\
\hline
\end{tabular}




\section{CP1n}

$(\mathrm{E}(\mathrm{RB}+\mathrm{HF}-\mathrm{LYP})=-1736.19576555 \quad$ A.U. $)$

\begin{tabular}{|c|c|c|c|c|c|}
\hline \multirow{2}{*}{$\begin{array}{l}\text { Center } \\
\text { Number }\end{array}$} & Atomic & Atomic & \multicolumn{3}{|c|}{ Coordinates } \\
\hline & Number & Type & $\mathrm{X}$ & $\mathrm{Y}$ & Z \\
\hline------1 & ------- & ------- & ---------- & ----------- & ---------- \\
\hline 1 & 6 & 0 & -1.467285 & 2.209451 & 1.269714 \\
\hline 2 & 1 & 0 & -1.584250 & 4.339310 & 1.348440 \\
\hline 3 & 6 & 0 & -2.075041 & 3.431453 & 1.038356 \\
\hline 4 & 6 & 0 & -3.345591 & 1.063363 & 0.245118 \\
\hline 5 & 6 & 0 & -3.311341 & 3.479646 & 0.411790 \\
\hline 6 & 6 & 0 & -2.102556 & 1.047410 & 0.874426 \\
\hline 7 & 6 & 0 & -3.933476 & 2.307482 & 0.021001 \\
\hline 8 & 1 & 0 & -4.884849 & 2.347919 & -0.483301 \\
\hline 9 & 1 & 0 & -3.787707 & 4.427416 & 0.222631 \\
\hline 10 & 6 & 0 & -3.343039 & -1.231076 & -0.764927 \\
\hline 11 & 6 & 0 & -4.028288 & -0.184258 & -0.152434 \\
\hline 12 & 6 & 0 & -5.335801 & -2.535863 & -0.933401 \\
\hline 13 & 6 & 0 & -5.395660 & -0.353736 & 0.060199 \\
\hline 14 & 6 & 0 & -3.976909 & -2.395285 & -1.153341 \\
\hline 15 & 6 & 0 & -6.046284 & -1.511796 & -0.325650 \\
\hline 16 & 1 & 0 & -7.102885 & -1.618210 & -0.143678 \\
\hline 17 & 1 & 0 & -5.945059 & 0.431385 & 0.552982 \\
\hline 18 & 1 & 0 & -5.834935 & -3.442579 & -1.232534 \\
\hline 19 & 8 & 0 & -2.005402 & -1.086065 & -1.077029 \\
\hline 20 & 8 & 0 & -1.507708 & -0.156043 & 1.189794 \\
\hline 21 & 15 & 0 & -0.887859 & -1.108191 & 0.060997 \\
\hline 22 & 8 & 0 & -0.561407 & -2.420850 & 0.628678 \\
\hline 23 & 8 & 0 & 0.235517 & -0.315006 & -0.671670 \\
\hline 24 & 1 & 0 & 1.181627 & -0.362003 & -0.346315 \\
\hline 25 & 1 & 0 & 4.668622 & -5.211296 & 0.183077 \\
\hline 26 & 6 & 0 & 4.328609 & -4.190478 & 0.119958 \\
\hline 27 & 6 & 0 & 3.433324 & -1.566409 & -0.038954 \\
\hline 28 & 6 & 0 & 3.286415 & -3.763270 & 0.927254 \\
\hline 29 & 6 & 0 & 4.931793 & -3.308884 & -0.762834 \\
\hline 30 & 6 & 0 & 4.482934 & -2.001012 & -0.840817 \\
\hline 31 & 6 & 0 & 2.829177 & -2.456881 & 0.852799 \\
\hline 32 & 1 & 0 & 2.819633 & -4.431582 & 1.631972 \\
\hline 33 & 1 & 0 & 5.736424 & -3.638742 & -1.399026 \\
\hline 34 & 1 & 0 & 4.913428 & -1.320117 & -1.557343 \\
\hline 35 & 7 & 0 & 2.894355 & -0.267284 & -0.141906 \\
\hline 36 & 6 & 0 & 3.683197 & 0.726796 & -0.148998 \\
\hline 37 & 1 & 0 & 4.749746 & 0.564135 & -0.009191 \\
\hline 38 & 6 & 0 & 3.285529 & 2.124821 & -0.310414 \\
\hline 39 & 6 & 0 & 2.656898 & 4.823863 & -0.570692 \\
\hline 40 & 6 & 0 & 4.267679 & 3.100909 & -0.152307 \\
\hline 41 & 6 & 0 & 1.979613 & 2.516681 & -0.610312 \\
\hline 42 & 6 & 0 & 1.672042 & 3.858235 & -0.736464 \\
\hline 43 & 6 & 0 & 3.957123 & 4.443837 & -0.279211 \\
\hline 44 & 1 & 0 & 5.279420 & 2.803530 & 0.075980 \\
\hline 45 & 1 & 0 & 1.212279 & 1.777294 & -0.753567 \\
\hline 46 & 1 & 0 & 0.660484 & 4.150216 & -0.966798 \\
\hline 47 & 1 & 0 & 4.725433 & 5.188637 & -0.152057 \\
\hline 48 & 1 & 0 & 2.410629 & 5.868418 & -0.672553 \\
\hline 49 & 8 & 0 & 1.857297 & -1.990207 & 1.671477 \\
\hline 50 & 1 & 0 & 1.003126 & -2.414108 & 1.475832 \\
\hline 51 & 1 & 0 & -3.396254 & -3.168082 & -1.626598 \\
\hline 52 & 1 & 0 & -0.512273 & 2.137683 & 1.761288 \\
\hline
\end{tabular}

TSt

$(\mathrm{E}(\mathrm{RB}+\mathrm{HF}-\mathrm{LYP})=-1736.19283370 \quad$ A.U. $)$ 


\begin{tabular}{|c|c|c|c|c|c|}
\hline \multirow{2}{*}{$\begin{array}{l}\text { Center } \\
\text { Number }\end{array}$} & Atomic & Atomic & \multicolumn{3}{|c|}{ Coordinates (Angstroms) } \\
\hline & Number & Type & $\mathrm{X}$ & $\mathrm{Y}$ & Z \\
\hline 1 & 6 & 0 & -1.793515 & 2.416153 & 1.162632 \\
\hline 2 & 1 & 0 & -2.129768 & 4.522569 & 1.058498 \\
\hline 3 & 6 & 0 & -2.497202 & 3.546775 & 0.785132 \\
\hline 4 & 6 & 0 & -3.455094 & 1.005385 & 0.098083 \\
\hline 5 & 6 & 0 & -3.674038 & 3.417977 & 0.062629 \\
\hline 6 & 6 & 0 & -2.272518 & 1.164724 & 0.818996 \\
\hline 7 & 6 & 0 & -4.141348 & 2.160308 & -0.274574 \\
\hline 8 & 1 & 0 & -5.046555 & 2.061668 & -0.850917 \\
\hline 9 & 1 & 0 & -4.224061 & 4.293467 & -0.240887 \\
\hline 10 & 6 & 0 & -3.141686 & -1.349061 & -0.700308 \\
\hline 11 & 6 & 0 & -3.977682 & -0.334471 & -0.236919 \\
\hline 12 & 6 & 0 & -4.978901 & -2.866319 & -0.891664 \\
\hline 13 & 6 & 0 & -5.334539 & -0.627771 & -0.109940 \\
\hline 14 & 6 & 0 & -3.627218 & -2.602045 & -1.024020 \\
\hline 15 & 6 & 0 & -5.835007 & -1.875793 & -0.434509 \\
\hline 16 & 1 & 0 & -6.887426 & -2.077342 & -0.321191 \\
\hline 17 & 1 & 0 & -5.996690 & 0.133815 & 0.268070 \\
\hline 18 & 1 & 0 & -5.359926 & -3.842854 & -1.141300 \\
\hline 19 & 8 & 0 & -1.810321 & -1.087901 & -0.929730 \\
\hline 20 & 8 & 0 & -1.595263 & 0.061060 & 1.277794 \\
\hline 21 & 15 & 0 & -0.769374 & -0.894676 & 0.278214 \\
\hline 22 & 8 & 0 & -0.413605 & -2.139085 & 0.983557 \\
\hline 23 & 8 & 0 & 0.345401 & -0.073173 & -0.364901 \\
\hline 24 & 1 & 0 & 1.492631 & -0.195646 & -0.184157 \\
\hline 25 & 1 & 0 & 4.617497 & -5.214050 & -0.042443 \\
\hline 26 & 6 & 0 & 4.253561 & -4.199809 & -0.066727 \\
\hline 27 & 6 & 0 & 3.306954 & -1.600786 & -0.115630 \\
\hline 28 & 6 & 0 & 3.364711 & -3.767302 & 0.903082 \\
\hline 29 & 6 & 0 & 4.673702 & -3.335249 & -1.065912 \\
\hline 30 & 6 & 0 & 4.197436 & -2.035941 & -1.088890 \\
\hline 31 & 6 & 0 & 2.874902 & -2.468579 & 0.890683 \\
\hline 32 & 1 & 0 & 3.039043 & -4.421329 & 1.694349 \\
\hline 33 & 1 & 0 & 5.356822 & -3.670594 & -1.828414 \\
\hline 34 & 1 & 0 & 4.485561 & -1.361553 & -1.879306 \\
\hline 35 & 7 & 0 & 2.788324 & -0.279015 & -0.140866 \\
\hline 36 & 6 & 0 & 3.590699 & 0.709220 & -0.198344 \\
\hline 37 & 1 & 0 & 4.652945 & 0.494180 & -0.159721 \\
\hline 38 & 6 & 0 & 3.245322 & 2.120835 & -0.294609 \\
\hline 39 & 6 & 0 & 2.765933 & 4.854960 & -0.443973 \\
\hline 40 & 6 & 0 & 4.299815 & 3.032064 & -0.215305 \\
\hline 41 & 6 & 0 & 1.940553 & 2.595254 & -0.458377 \\
\hline 42 & 6 & 0 & 1.710732 & 3.955902 & -0.530787 \\
\hline 43 & 6 & 0 & 4.063231 & 4.392608 & -0.286625 \\
\hline 44 & 1 & 0 & 5.308308 & 2.669105 & -0.092614 \\
\hline 45 & 1 & 0 & 1.116536 & 1.908237 & -0.534530 \\
\hline 46 & 1 & 0 & 0.702370 & 4.314224 & -0.657182 \\
\hline 47 & 1 & 0 & 4.884354 & 5.086912 & -0.221130 \\
\hline 48 & 1 & 0 & 2.576356 & 5.914507 & -0.502148 \\
\hline 49 & 8 & 0 & 2.070712 & -2.017527 & 1.874988 \\
\hline 50 & 1 & 0 & 1.125958 & -2.177715 & 1.655117 \\
\hline 51 & 1 & 0 & -2.936527 & -3.346901 & -1.379741 \\
\hline 52 & 1 & 0 & -0.883304 & 2.481312 & 1.734087 \\
\hline
\end{tabular}

\section{TS1i}

\section{$(\mathrm{E}(\mathrm{RB}+\mathrm{HF}-\mathrm{LYP})=-2412.98377768 \quad$ A.U. $)$}

Center Atomic Atomic Coordinates (Angstroms)




\begin{tabular}{|c|c|c|c|c|c|}
\hline Number & Number & Type & X & Y & Z \\
\hline 1 & 6 & 0 & 3.385033 & 2.593944 & -1.428069 \\
\hline 2 & 1 & 0 & 4.003271 & 4.630526 & -1.600205 \\
\hline 3 & 6 & 0 & 4.303266 & 3.625856 & -1.349510 \\
\hline 4 & 6 & 0 & 5.070052 & 1.012449 & -0.700026 \\
\hline 5 & 6 & 0 & 5.603932 & 3.363166 & -0.946718 \\
\hline 6 & 6 & 0 & 3.766977 & 1.302344 & -1.108619 \\
\hline 7 & 6 & 0 & 5.975057 & 2.069849 & -0.624761 \\
\hline 8 & 1 & 0 & 6.979956 & 1.868136 & -0.291129 \\
\hline 9 & 1 & 0 & 6.323690 & 4.162225 & -0.875395 \\
\hline 10 & 6 & 0 & 4.666892 & -1.208267 & 0.379900 \\
\hline 11 & 6 & 0 & 5.483104 & -0.367742 & -0.377836 \\
\hline 12 & 6 & 0 & 6.280023 & -2.973594 & 0.259963 \\
\hline 13 & 6 & 0 & 6.710330 & -0.870787 & -0.806416 \\
\hline 14 & 6 & 0 & 5.055568 & -2.498465 & 0.694452 \\
\hline 15 & 6 & 0 & 7.111400 & -2.156798 & -0.491873 \\
\hline 16 & 1 & 0 & 8.063273 & -2.522930 & -0.840444 \\
\hline 17 & 1 & 0 & 7.345721 & -0.243539 & -1.410382 \\
\hline 18 & 1 & 0 & 6.581524 & -3.979111 & 0.504761 \\
\hline 19 & 8 & 0 & 3.495936 & -0.731225 & 0.902101 \\
\hline 20 & 8 & 0 & 2.863372 & 0.292353 & -1.287002 \\
\hline 21 & 15 & 0 & 2.187787 & -0.485128 & -0.027724 \\
\hline 22 & 8 & 0 & 1.653886 & -1.769609 & -0.556710 \\
\hline 23 & 8 & 0 & 1.289386 & 0.423975 & 0.738885 \\
\hline 24 & 1 & 0 & -0.213589 & -0.190024 & 1.324310 \\
\hline 25 & 1 & 0 & -2.520380 & -5.547162 & 1.817227 \\
\hline 26 & 6 & 0 & -2.247271 & -4.506414 & 1.748841 \\
\hline 27 & 6 & 0 & -1.530653 & -1.830867 & 1.552438 \\
\hline 28 & 6 & 0 & -1.682023 & -4.021676 & 0.582882 \\
\hline 29 & 6 & 0 & -2.457862 & -3.659090 & 2.826442 \\
\hline 30 & 6 & 0 & -2.091685 & -2.328730 & 2.722961 \\
\hline 31 & 6 & 0 & -1.316963 & -2.684171 & 0.463290 \\
\hline 32 & 1 & 0 & -1.512650 & -4.662792 & -0.265926 \\
\hline 33 & 1 & 0 & -2.890615 & -4.030704 & 3.740445 \\
\hline 34 & 1 & 0 & -2.224651 & -1.654676 & 3.553697 \\
\hline 35 & 7 & 0 & -1.195092 & -0.448978 & 1.497223 \\
\hline 36 & 6 & 0 & -2.138122 & 0.469279 & 1.272903 \\
\hline 37 & 1 & 0 & -3.139331 & 0.083340 & 1.383990 \\
\hline 38 & 6 & 0 & -1.985579 & 1.874270 & 1.706741 \\
\hline 39 & 6 & 0 & -1.840015 & 4.504843 & 2.596829 \\
\hline 40 & 6 & 0 & -3.143098 & 2.575927 & 2.033403 \\
\hline 41 & 6 & 0 & -0.745988 & 2.500330 & 1.827276 \\
\hline 42 & 6 & 0 & -0.683782 & 3.810609 & 2.269369 \\
\hline 43 & 6 & 0 & -3.073441 & 3.884316 & 2.480288 \\
\hline 44 & 1 & 0 & -4.103734 & 2.089375 & 1.951641 \\
\hline 45 & 1 & 0 & 0.157884 & 1.972316 & 1.569717 \\
\hline 46 & 1 & 0 & 0.276992 & 4.289961 & 2.361151 \\
\hline 47 & 1 & 0 & -3.975885 & 4.412858 & 2.741682 \\
\hline 48 & 1 & 0 & -1.778867 & 5.523394 & 2.944580 \\
\hline 49 & 8 & 0 & -0.858190 & -2.228349 & -0.718679 \\
\hline 50 & 1 & 0 & 0.127924 & -2.035255 & -0.689816 \\
\hline 51 & 1 & 0 & 4.388347 & -3.106039 & 1.281106 \\
\hline 52 & 1 & 0 & 2.369866 & 2.764039 & -1.743434 \\
\hline 53 & 6 & 0 & -2.155533 & 0.673657 & -0.793979 \\
\hline 54 & 6 & 0 & -3.355237 & 1.251160 & -1.150121 \\
\hline 55 & 8 & 0 & -4.462116 & 0.617452 & -1.415649 \\
\hline 56 & 14 & 0 & -4.852555 & -1.013477 & -1.842494 \\
\hline 57 & 6 & 0 & -6.486435 & -0.781088 & -2.711967 \\
\hline 58 & 6 & 0 & -5.058558 & -1.971002 & -0.256877 \\
\hline 59 & 6 & 0 & -3.544281 & -1.701455 & -2.974952 \\
\hline 60 & 1 & 0 & -1.989947 & -0.360521 & -1.034094 \\
\hline 61 & 1 & 0 & -1.287702 & 1.307280 & -0.840982 \\
\hline 62 & 8 & 0 & -3.451829 & 2.559649 & -1.125976 \\
\hline 63 & 1 & 0 & -6.903734 & -1.741602 & -3.009005 \\
\hline
\end{tabular}




$\begin{array}{lllrrr}64 & 1 & 0 & -6.377219 & -0.177664 & -3.610443 \\ 65 & 1 & 0 & -7.212567 & -0.292302 & -2.065985 \\ 66 & 1 & 0 & -5.430675 & -2.970740 & -0.476966 \\ 67 & 1 & 0 & -5.780753 & -1.493094 & 0.402259 \\ 68 & 1 & 0 & -4.124231 & -2.093069 & 0.284253 \\ 69 & 1 & 0 & -3.279884 & -0.991326 & -3.756233 \\ 70 & 1 & 0 & -3.939045 & -2.589563 & -3.467830 \\ 71 & 1 & 0 & -2.633943 & -1.990717 & -2.455736 \\ 72 & 6 & 0 & -4.711396 & 3.182183 & -1.335882 \\ 73 & 1 & 0 & -5.091745 & 2.954026 & -2.324785 \\ 74 & 1 & 0 & -4.520090 & 4.241701 & -1.241865 \\ 75 & 1 & 0 & -5.428417 & 2.863925 & -0.587937 \\ -\end{array}$

\section{CP2i}

\section{$(\mathrm{E}(\mathrm{RB}+\mathrm{HF}-\mathrm{LYP})=-1736.19240951 \quad$ A.U. $)$}

\begin{tabular}{|c|c|c|c|c|c|}
\hline \multirow{2}{*}{$\begin{array}{l}\text { Center } \\
\text { Number }\end{array}$} & Atomic & Atomic & \multicolumn{3}{|c|}{ Coordinates (Angstroms) } \\
\hline & Number & Type & $\mathrm{X}$ & Y & Z \\
\hline \multicolumn{6}{|c|}{$----------------------1+---------------------------------------------$} \\
\hline 1 & 6 & 0 & -3.131743 & 2.537600 & -1.283905 \\
\hline 2 & 1 & 0 & -4.602706 & 3.676487 & -2.331364 \\
\hline 3 & 6 & 0 & -4.408032 & 2.785069 & -1.757630 \\
\hline 4 & 6 & 0 & -3.887645 & 0.464850 & -0.279882 \\
\hline 5 & 6 & 0 & -5.428193 & 1.882462 & -1.497998 \\
\hline 6 & 6 & 0 & -2.880510 & 1.392089 & -0.550352 \\
\hline 7 & 6 & 0 & -5.163809 & 0.736156 & -0.769967 \\
\hline 8 & 1 & 0 & -5.950787 & 0.022223 & -0.589223 \\
\hline 9 & 1 & 0 & -6.422917 & 2.064187 & -1.870708 \\
\hline 10 & 6 & 0 & -2.469747 & -1.515551 & 0.294740 \\
\hline 11 & 6 & 0 & -3.616946 & -0.752027 & 0.511107 \\
\hline 12 & 6 & 0 & -3.111203 & -3.069566 & 1.997781 \\
\hline 13 & 6 & 0 & -4.506135 & -1.186844 & 1.492331 \\
\hline 14 & 6 & 0 & -2.211750 & -2.659183 & 1.029503 \\
\hline 15 & 6 & 0 & -4.263632 & -2.333349 & 2.227839 \\
\hline 16 & 1 & 0 & -4.965486 & -2.644082 & 2.984380 \\
\hline 17 & 1 & 0 & -5.389447 & -0.600844 & 1.687778 \\
\hline 18 & 1 & 0 & -2.910112 & -3.959463 & 2.571664 \\
\hline 19 & 8 & 0 & -1.608135 & -1.183632 & -0.718669 \\
\hline 20 & 8 & 0 & -1.630203 & 1.216837 & -0.014266 \\
\hline 21 & 15 & 0 & -0.617413 & 0.092270 & -0.606843 \\
\hline 22 & 8 & 0 & 0.341722 & -0.149337 & 0.554864 \\
\hline 23 & 8 & 0 & -0.054408 & 0.415960 & -1.920674 \\
\hline 24 & 1 & 0 & 1.842692 & -0.058096 & 0.193588 \\
\hline 25 & 1 & 0 & 4.205594 & -5.125631 & -1.353925 \\
\hline 26 & 6 & 0 & 3.930259 & -4.118212 & -1.088363 \\
\hline 27 & 6 & 0 & 3.207959 & -1.542559 & -0.389578 \\
\hline 28 & 6 & 0 & 2.860822 & -3.907753 & -0.245637 \\
\hline 29 & 6 & 0 & 4.635511 & -3.042262 & -1.617846 \\
\hline 30 & 6 & 0 & 4.265001 & -1.762473 & -1.269842 \\
\hline 31 & 6 & 0 & 2.461448 & -2.620119 & 0.112579 \\
\hline 32 & 1 & 0 & 2.293377 & -4.728689 & 0.157746 \\
\hline 33 & 1 & 0 & 5.445926 & -3.201258 & -2.308330 \\
\hline 34 & 1 & 0 & 4.771430 & -0.918228 & -1.708431 \\
\hline 35 & 7 & 0 & 2.873704 & -0.225651 & 0.015362 \\
\hline 36 & 6 & 0 & 3.714354 & 0.732305 & 0.161960 \\
\hline 37 & 1 & 0 & 4.753223 & 0.495120 & -0.010713 \\
\hline 38 & 6 & 0 & 3.409589 & 2.089657 & 0.545161 \\
\hline 39 & 8 & 0 & 1.435647 & -2.511648 & 0.956848 \\
\hline 40 & 1 & 0 & 0.950141 & -1.657368 & 0.891276 \\
\hline 41 & 1 & 0 & -1.306308 & -3.206950 & 0.830105 \\
\hline 42 & 1 & 0 & -2.318728 & 3.218332 & -1.469056 \\
\hline
\end{tabular}




\begin{tabular}{llllll}
43 & 6 & 0 & 4.480477 & 2.988145 & 0.584966 \\
44 & 6 & 0 & 4.274675 & 4.306041 & 0.942276 \\
45 & 6 & 0 & 2.996318 & 4.734123 & 1.269375 \\
46 & 6 & 0 & 1.927659 & 3.846386 & 1.240739 \\
47 & 6 & 0 & 2.122112 & 2.528304 & 0.880576 \\
48 & 1 & 0 & 5.472111 & 2.648630 & 0.330155 \\
49 & 1 & 0 & 5.102441 & 4.994582 & 0.966981 \\
50 & 1 & 0 & 2.831182 & 5.761719 & 1.549310 \\
51 & 1 & 0 & 0.937723 & 4.182755 & 1.498548 \\
52 & 1 & 0 & 1.287909 & 1.846913 & 0.870915 \\
\hline
\end{tabular}

\section{CP2n}

\section{$(\mathrm{E}(\mathrm{RB}+\mathrm{HF}-\mathrm{LYP})=-1736.19132184 \quad$ A.U. $)$}

\begin{tabular}{|c|c|c|c|c|c|}
\hline \multirow{2}{*}{$\begin{array}{l}\text { Center } \\
\text { Number }\end{array}$} & Atomic & Atomic & \multicolumn{2}{|c|}{ Coordinates } & (Angstroms) \\
\hline & Number & Type & $\mathrm{X}$ & $\mathrm{Y}$ & Z \\
\hline & ------ & ----1 & -ーーーー-ーー- & ---------- & ---------- \\
\hline 1 & 6 & 0 & -3.408663 & 2.574704 & -1.468078 \\
\hline 2 & 1 & 0 & -5.001224 & 3.557846 & -2.494417 \\
\hline 3 & 6 & 0 & -4.728724 & 2.739460 & -1.848783 \\
\hline 4 & 6 & 0 & -4.009996 & 0.607940 & -0.178466 \\
\hline 5 & 6 & 0 & -5.692077 & 1.847306 & -1.402565 \\
\hline 6 & 6 & 0 & -3.066046 & 1.521140 & -0.642647 \\
\hline 7 & 6 & 0 & -5.331789 & 0.795097 & -0.580123 \\
\hline 8 & 1 & 0 & -6.077820 & 0.089672 & -0.253182 \\
\hline 9 & 1 & 0 & -6.720285 & 1.964537 & -1.702577 \\
\hline 10 & 6 & 0 & -2.483292 & -1.257766 & 0.508460 \\
\hline 11 & 6 & 0 & -3.633856 & -0.500971 & 0.720418 \\
\hline 12 & 6 & 0 & -2.932625 & -2.610221 & 2.424279 \\
\hline 13 & 6 & 0 & -4.427404 & -0.836652 & 1.816429 \\
\hline 14 & 6 & 0 & -2.124834 & -2.298207 & 1.344534 \\
\hline 15 & 6 & 0 & -4.087920 & -1.880095 & 2.658626 \\
\hline 16 & 1 & 0 & -4.717105 & -2.114392 & 3.501422 \\
\hline 17 & 1 & 0 & -5.311522 & -0.253146 & 2.014120 \\
\hline 18 & 1 & 0 & -2.656671 & -3.418965 & 3.080632 \\
\hline 19 & 8 & 0 & -1.704758 & -1.031451 & -0.609270 \\
\hline 20 & 8 & 0 & -1.756510 & 1.428709 & -0.212436 \\
\hline 21 & 15 & 0 & -0.800603 & 0.275201 & -0.779239 \\
\hline 22 & 8 & 0 & 0.266156 & 0.223793 & 0.384378 \\
\hline 23 & 8 & 0 & -0.281677 & 0.424708 & -2.130599 \\
\hline 24 & 1 & 0 & 1.213788 & 0.141203 & 0.079433 \\
\hline 25 & 1 & 0 & 3.256862 & -5.382634 & -1.613780 \\
\hline 26 & 6 & 0 & 3.179419 & -4.354259 & -1.301118 \\
\hline 27 & 6 & 0 & 2.965095 & -1.705081 & -0.476180 \\
\hline 28 & 6 & 0 & 2.297985 & -4.012788 & -0.293153 \\
\hline 29 & 6 & 0 & 3.942351 & -3.376241 & -1.922588 \\
\hline 30 & 6 & 0 & 3.827724 & -2.062722 & -1.509972 \\
\hline 31 & 6 & 0 & 2.169336 & -2.693238 & 0.116607 \\
\hline 32 & 1 & 0 & 1.686757 & -4.754157 & 0.193010 \\
\hline 33 & 1 & 0 & 4.606527 & -3.630457 & -2.731462 \\
\hline 34 & 1 & 0 & 4.384416 & -1.288838 & -2.012672 \\
\hline 35 & 7 & 0 & 2.835991 & -0.372800 & -0.028462 \\
\hline 36 & 6 & 0 & 3.878840 & 0.346352 & 0.085264 \\
\hline 37 & 1 & 0 & 4.852507 & -0.090378 & -0.121657 \\
\hline 38 & 6 & 0 & 3.909017 & 1.747486 & 0.493788 \\
\hline 39 & 8 & 0 & 1.300522 & -2.443019 & 1.119758 \\
\hline 40 & 1 & 0 & 1.083560 & -1.508220 & 1.175616 \\
\hline 41 & 1 & 0 & -1.217793 & -2.840776 & 1.140040 \\
\hline 42 & 1 & 0 & -2.636507 & 3.249628 & -1.794720 \\
\hline 43 & 6 & 0 & 5.147763 & 2.387466 & 0.501959 \\
\hline 44 & 6 & 0 & 5.256268 & 3.714650 & 0.878385 \\
\hline
\end{tabular}




\begin{tabular}{llllll}
45 & 6 & 0 & 4.122745 & 4.415423 & 1.257041 \\
46 & 6 & 0 & 2.884491 & 3.785485 & 1.258858 \\
47 & 6 & 0 & 2.772808 & 2.461847 & 0.880046 \\
48 & 1 & 0 & 6.029604 & 1.839636 & 0.208250 \\
49 & 1 & 0 & 6.219250 & 4.197967 & 0.877193 \\
50 & 1 & 0 & 4.201173 & 5.449080 & 1.552285 \\
51 & 1 & 0 & 2.003320 & 4.329338 & 1.556664 \\
52 & 1 & 0 & 1.808708 & 1.986022 & 0.892851 \\
\hline
\end{tabular}

\section{TS2i}

\section{$(\mathrm{E}(\mathrm{RB}+\mathrm{HF}-\mathrm{LYP})=-2412.97838563 \quad$ A.U. $)$}

\begin{tabular}{|c|c|c|c|c|c|}
\hline \multirow{2}{*}{$\begin{array}{l}\text { Center } \\
\text { Number }\end{array}$} & \multirow{2}{*}{$\begin{array}{l}\text { Atomic } \\
\text { Number }\end{array}$} & \multirow{2}{*}{$\begin{array}{c}\text { Atomic } \\
\text { Type }\end{array}$} & \multicolumn{3}{|c|}{ Coordinates (Angstroms) } \\
\hline & & & $\mathrm{X}$ & Y & Z \\
\hline$-5-2$ & ----- & ------ & ---------- & ---------- & ---------- \\
\hline 1 & 6 & 0 & -5.144164 & 0.432266 & -1.056699 \\
\hline 2 & 1 & 0 & -7.131623 & 1.210889 & -1.090380 \\
\hline 3 & 6 & 0 & -6.291266 & 0.939247 & -0.472369 \\
\hline 4 & 6 & 0 & -4.113901 & 0.207654 & 1.124360 \\
\hline 5 & 6 & 0 & -6.359235 & 1.086618 & 0.905077 \\
\hline 6 & 6 & 0 & -4.065995 & 0.077799 & -0.264520 \\
\hline 7 & 6 & 0 & -5.280518 & 0.719299 & 1.689609 \\
\hline 8 & 1 & 0 & -5.343425 & 0.805889 & 2.762282 \\
\hline 9 & 1 & 0 & -7.253432 & 1.471672 & 1.367372 \\
\hline 10 & 6 & 0 & -2.258529 & -1.360879 & 1.730448 \\
\hline 11 & 6 & 0 & -2.962255 & -0.175776 & 1.964733 \\
\hline 12 & 6 & 0 & -0.820032 & -0.930544 & 3.600842 \\
\hline 13 & 6 & 0 & -2.558831 & 0.618378 & 3.037137 \\
\hline 14 & 6 & 0 & -1.194865 & -1.733514 & 2.537371 \\
\hline 15 & 6 & 0 & -1.502975 & 0.250523 & 3.852361 \\
\hline 16 & 1 & 0 & -1.216427 & 0.881063 & 4.678612 \\
\hline 17 & 1 & 0 & -3.081827 & 1.542964 & 3.221213 \\
\hline 18 & 1 & 0 & -0.002252 & -1.232464 & 4.235856 \\
\hline 19 & 8 & 0 & -2.667101 & -2.220869 & 0.755476 \\
\hline 20 & 8 & 0 & -2.907967 & -0.329890 & -0.863963 \\
\hline 21 & 15 & 0 & -2.453559 & -1.909454 & -0.834229 \\
\hline 22 & 8 & 0 & -0.967916 & -1.817028 & -1.094569 \\
\hline 23 & 8 & 0 & -3.333925 & -2.774435 & -1.618196 \\
\hline 24 & 1 & 0 & 0.491711 & -1.392125 & -1.987801 \\
\hline 25 & 1 & 0 & 5.111785 & -4.530962 & -0.320135 \\
\hline 26 & 6 & 0 & 4.363565 & -3.832423 & -0.658351 \\
\hline 27 & 6 & 0 & 2.417807 & -2.050170 & -1.510571 \\
\hline 28 & 6 & 0 & 3.133496 & -3.799533 & -0.031867 \\
\hline 29 & 6 & 0 & 4.633051 & -2.986008 & -1.725993 \\
\hline 30 & 6 & 0 & 3.652612 & -2.108693 & -2.147377 \\
\hline 31 & 6 & 0 & 2.136368 & -2.913790 & -0.441887 \\
\hline 32 & 1 & 0 & 2.905256 & -4.455874 & 0.790888 \\
\hline 33 & 1 & 0 & 5.583075 & -3.022208 & -2.232008 \\
\hline 34 & 1 & 0 & 3.822526 & -1.459860 & -2.991952 \\
\hline 35 & 7 & 0 & 1.465594 & -1.091876 & -1.946847 \\
\hline 36 & 6 & 0 & 1.686223 & 0.213415 & -1.785963 \\
\hline 37 & 1 & 0 & 2.725961 & 0.466017 & -1.656019 \\
\hline 38 & 6 & 0 & 0.877893 & 1.223435 & -2.488397 \\
\hline 39 & 8 & 0 & 0.994973 & -2.909575 & 0.248633 \\
\hline 40 & 1 & 0 & 0.225397 & -2.516077 & -0.231756 \\
\hline 41 & 1 & 0 & -0.681868 & -2.654036 & 2.318508 \\
\hline 42 & 1 & 0 & -5.063957 & 0.300228 & -2.122072 \\
\hline 43 & 6 & 0 & 1.150505 & 0.530887 & 0.242710 \\
\hline 44 & 6 & 0 & 1.526646 & 1.820441 & 0.534860 \\
\hline 45 & 8 & 0 & 2.696796 & 2.190443 & 0.989203 \\
\hline 46 & 14 & 0 & 3.851071 & 1.381844 & 1.981677 \\
\hline
\end{tabular}




\begin{tabular}{|c|c|c|c|c|c|}
\hline 47 & 6 & 0 & 4.703828 & 0.051154 & 0.992013 \\
\hline 48 & 6 & 0 & 2.939366 & 0.722465 & 3.468597 \\
\hline 49 & 6 & 0 & 5.024479 & 2.763861 & 2.413081 \\
\hline 50 & 1 & 0 & 1.722454 & -0.273388 & 0.668664 \\
\hline 51 & 1 & 0 & 0.101683 & 0.342221 & 0.096844 \\
\hline 52 & 8 & 0 & 0.706749 & 2.796065 & 0.232594 \\
\hline 53 & 1 & 0 & 5.489962 & 3.180797 & 1.522624 \\
\hline 54 & 1 & 0 & 5.820558 & 2.405085 & 3.063080 \\
\hline 55 & 1 & 0 & 4.513067 & 3.571191 & 2.932268 \\
\hline 56 & 1 & 0 & 2.523386 & 1.532477 & 4.064208 \\
\hline 57 & 6 & 0 & 1.516782 & 2.382121 & -2.922238 \\
\hline 58 & 6 & 0 & 0.812779 & 3.355514 & -3.610805 \\
\hline 59 & 6 & 0 & -0.540311 & 3.181198 & -3.856528 \\
\hline 60 & 1 & 0 & 3.616757 & 0.158156 & 4.107507 \\
\hline 61 & 1 & 0 & 2.120609 & 0.063037 & 3.191045 \\
\hline 62 & 1 & 0 & 5.095864 & 0.441659 & 0.054905 \\
\hline 63 & 1 & 0 & 4.065155 & -0.796753 & 0.759824 \\
\hline 64 & 1 & 0 & 5.552222 & -0.331108 & 1.558585 \\
\hline 65 & 6 & 0 & -1.183439 & 2.033973 & -3.413136 \\
\hline 66 & 6 & 0 & -0.482809 & 1.054187 & -2.733628 \\
\hline 67 & 1 & 0 & 2.571620 & 2.515149 & -2.734217 \\
\hline 68 & 1 & 0 & 1.318853 & 4.241993 & -3.956992 \\
\hline 69 & 1 & 0 & -1.093019 & 3.937490 & -4.389864 \\
\hline 70 & 1 & 0 & -2.237503 & 1.899640 & -3.589547 \\
\hline 71 & 1 & 0 & -0.997848 & 0.181144 & -2.372710 \\
\hline 72 & 6 & 0 & 1.115298 & 4.147062 & 0.394383 \\
\hline 73 & 1 & 0 & 0.273186 & 4.735882 & 0.060040 \\
\hline 74 & 1 & 0 & 1.986580 & 4.361435 & -0.212947 \\
\hline 75 & 1 & 0 & 1.332414 & 4.359193 & 1.435024 \\
\hline
\end{tabular}

\section{TS3r}

\section{$(\mathrm{E}(\mathrm{RB}+\mathrm{HF}-\mathrm{LYP})=-3283.59740201 \quad$ A.U. $)$}

\begin{tabular}{|c|c|c|c|c|c|}
\hline \multirow{2}{*}{$\begin{array}{l}\text { Center } \\
\text { Number }\end{array}$} & Atomic & Atomic & \multicolumn{3}{|c|}{ Coordinates (Angstroms) } \\
\hline & Number & Type & $\mathrm{X}$ & Y & Z \\
\hline------ & ------ & ------- & ---------- & ---------- & ----------- \\
\hline 1 & 6 & 0 & 0.255871 & 4.508737 & -0.156848 \\
\hline 2 & 1 & 0 & -0.424849 & 6.398748 & 0.565613 \\
\hline 3 & 6 & 0 & 0.439347 & 5.788294 & 0.360458 \\
\hline 4 & 6 & 0 & 2.679078 & 4.226126 & -0.205878 \\
\hline 5 & 6 & 0 & 1.708307 & 6.289665 & 0.588224 \\
\hline 6 & 6 & 0 & 1.388935 & 3.742559 & -0.435170 \\
\hline 7 & 6 & 0 & 2.817298 & 5.511939 & 0.307678 \\
\hline 8 & 1 & 0 & 3.806476 & 5.892981 & 0.501638 \\
\hline 9 & 1 & 0 & 1.832195 & 7.283456 & 0.985375 \\
\hline 10 & 6 & 0 & 3.917036 & 2.055747 & -0.163492 \\
\hline 11 & 6 & 0 & 3.865435 & 3.401403 & -0.526293 \\
\hline 12 & 6 & 0 & 6.067948 & 1.820339 & -1.174583 \\
\hline 13 & 6 & 0 & 4.955706 & 3.942462 & -1.201272 \\
\hline 14 & 6 & 0 & 5.001931 & 1.243376 & -0.491203 \\
\hline 15 & 6 & 0 & 6.052151 & 3.161024 & -1.518216 \\
\hline 16 & 1 & 0 & 6.888250 & 3.592372 & -2.043268 \\
\hline 17 & 1 & 0 & 4.927166 & 4.978098 & -1.498024 \\
\hline 18 & 1 & 0 & 6.919780 & 1.209458 & -1.425353 \\
\hline 19 & 8 & 0 & 2.889779 & 1.537455 & 0.570099 \\
\hline 20 & 8 & 0 & 1.233261 & 2.514468 & -1.004630 \\
\hline 21 & 15 & 0 & 1.483805 & 1.141529 & -0.155010 \\
\hline 22 & 8 & 0 & 1.684827 & 0.070789 & -1.162272 \\
\hline 23 & 8 & 0 & 0.487471 & 0.995252 & 0.938400 \\
\hline 24 & 1 & 0 & -0.055221 & -0.613548 & 1.347291 \\
\hline 25 & 1 & 0 & 2.314558 & -5.818779 & 0.223349 \\
\hline
\end{tabular}




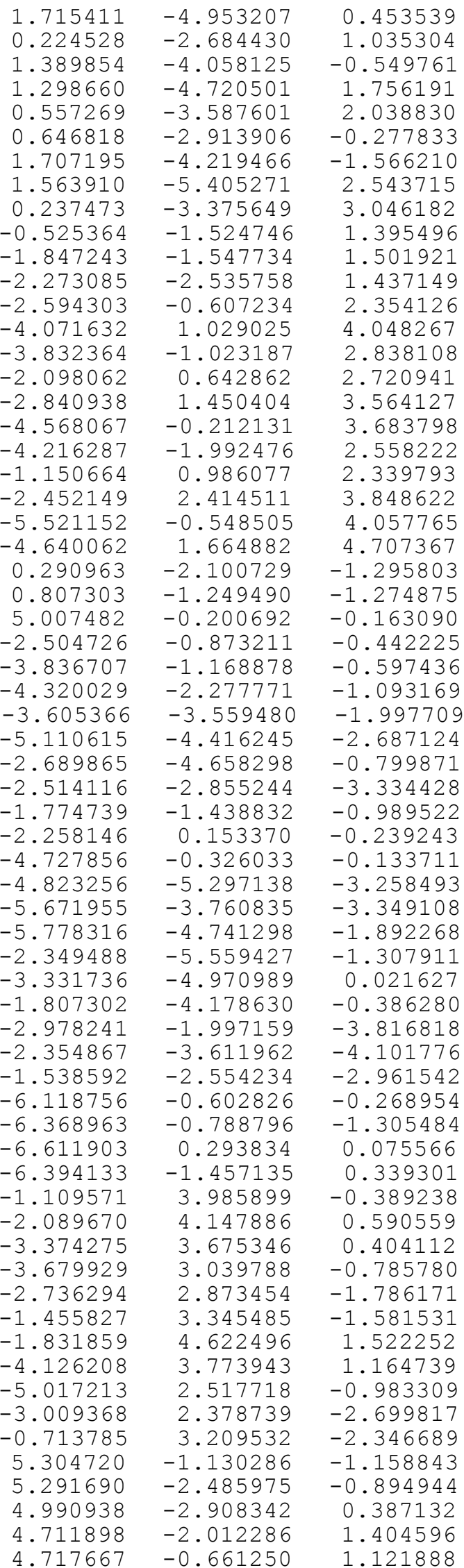




$\begin{array}{rrrrrr}91 & 1 & 0 & 5.508224 & -0.787122 & -2.158965 \\ 92 & 1 & 0 & 5.492707 & -3.210282 & -1.662493 \\ 93 & 7 & 0 & 4.941755 & -4.330822 & 0.668114 \\ 94 & 1 & 0 & 4.483461 & -2.379146 & 2.388045 \\ 95 & 1 & 0 & 4.481875 & 0.044062 & 1.898358 \\ 96 & 8 & 0 & -5.257295 & 1.943513 & -2.023411 \\ 97 & 8 & 0 & -5.826716 & 2.676316 & -0.093986 \\ 98 & 8 & 0 & 4.782123 & -4.677859 & 1.817397 \\ 99 & 8 & 0 & 5.052405 & -5.096811 & -0.265731 \\ -\end{array}$

\section{TS3s}

$(\mathrm{E}(\mathrm{RB}+\mathrm{HF}-\mathrm{LYP})=-3283.58834293 \quad$ A.U. $)$

\begin{tabular}{|c|c|c|c|c|c|}
\hline \multirow{2}{*}{$\begin{array}{l}\text { Center } \\
\text { Number }\end{array}$} & Atomic & Atomic & \multicolumn{3}{|c|}{ Coordinates (Angstroms) } \\
\hline & Number & Type & $\mathrm{x}$ & $Y$ & Z \\
\hline 1 & 6 & 0 & 4.406306 & 1.485000 & -1.162421 \\
\hline 2 & 1 & 0 & 5.999470 & 1.145043 & -2.541518 \\
\hline 3 & 6 & 0 & 5.129333 & 1.742582 & -2.325591 \\
\hline 4 & 6 & 0 & 2.915974 & 3.324971 & -1.740924 \\
\hline 5 & 6 & 0 & 4.768231 & 2.762843 & -3.184828 \\
\hline 6 & 6 & 0 & 3.301720 & 2.293851 & -0.878422 \\
\hline 7 & 6 & 0 & 3.664671 & 3.542407 & -2.893366 \\
\hline 8 & 1 & 0 & 3.364236 & 4.326257 & -3.569079 \\
\hline 9 & 1 & 0 & 5.342899 & 2.947800 & -4.077192 \\
\hline 10 & 6 & 0 & 0.543510 & 3.620662 & -1.024259 \\
\hline 11 & 6 & 0 & 1.746380 & 4.181334 & -1.448002 \\
\hline 12 & 6 & 0 & -0.485504 & 5.777310 & -0.949548 \\
\hline 13 & 6 & 0 & 1.804352 & 5.562028 & -1.619450 \\
\hline 14 & 6 & 0 & -0.584710 & 4.401232 & -0.774428 \\
\hline 15 & 6 & 0 & 0.699691 & 6.355702 & -1.370573 \\
\hline 16 & 1 & 0 & 0.761646 & 7.423238 & -1.502223 \\
\hline 17 & 1 & 0 & 2.732595 & 6.012368 & -1.930554 \\
\hline 18 & 1 & 0 & -1.351702 & 6.392515 & -0.767712 \\
\hline 19 & 8 & 0 & 0.463926 & 2.265244 & -0.887705 \\
\hline 20 & 8 & 0 & 2.648399 & 2.129763 & 0.302000 \\
\hline 21 & 15 & 0 & 1.111360 & 1.578646 & 0.452147 \\
\hline 22 & 8 & 0 & 0.572295 & 2.221843 & 1.678072 \\
\hline 23 & 8 & 0 & 1.030915 & 0.111465 & 0.249311 \\
\hline 24 & 1 & 0 & 0.000464 & -1.083509 & 1.752901 \\
\hline 25 & 1 & 0 & -4.464386 & 1.368316 & 4.292576 \\
\hline 26 & 6 & 0 & -3.747707 & 0.754384 & 3.773227 \\
\hline 27 & 6 & 0 & -1.901279 & -0.810649 & 2.410859 \\
\hline 28 & 6 & 0 & -2.390463 & 0.997657 & 3.916436 \\
\hline 29 & 6 & 0 & -4.183097 & -0.277126 & 2.957045 \\
\hline 30 & 6 & 0 & -3.262134 & -1.057769 & 2.276986 \\
\hline 31 & 6 & 0 & -1.458092 & 0.225303 & 3.244232 \\
\hline 32 & 1 & 0 & -2.027564 & 1.787052 & 4.553023 \\
\hline 33 & 1 & 0 & -5.236286 & -0.463151 & 2.830828 \\
\hline 34 & 1 & 0 & -3.619379 & -1.849445 & 1.640461 \\
\hline 35 & 7 & 0 & -0.902223 & -1.544282 & 1.754852 \\
\hline 36 & 6 & 0 & -1.112665 & -2.525585 & 0.884291 \\
\hline 37 & 1 & 0 & -2.060817 & -3.021383 & 0.993721 \\
\hline 38 & 6 & 0 & -0.018015 & -3.417437 & 0.465602 \\
\hline 39 & 6 & 0 & 1.964571 & -5.232560 & -0.242895 \\
\hline 40 & 6 & 0 & -0.322803 & -4.767328 & 0.298509 \\
\hline 41 & 6 & 0 & 1.286289 & -2.974525 & 0.254557 \\
\hline 42 & 6 & 0 & 2.268013 & -3.886954 & -0.092775 \\
\hline 43 & 6 & 0 & 0.663971 & -5.673352 & -0.051208 \\
\hline 44 & 1 & 0 & -1.331259 & -5.113641 & 0.466247 \\
\hline 45 & 1 & 0 & 1.520885 & -1.925037 & 0.329307 \\
\hline
\end{tabular}




\begin{tabular}{|c|c|c|c|c|c|}
\hline 46 & 1 & 0 & 3.276880 & -3.541399 & -0.244632 \\
\hline 47 & 1 & 0 & 0.421648 & -6.718231 & -0.157559 \\
\hline 48 & 1 & 0 & 2.739708 & -5.934481 & -0.504254 \\
\hline 49 & 8 & 0 & -0.131525 & 0.406192 & 3.393790 \\
\hline 50 & 1 & 0 & 0.171070 & 1.181040 & 2.850898 \\
\hline 51 & 6 & 0 & -1.854758 & 3.784247 & -0.334590 \\
\hline 52 & 6 & 0 & -1.682246 & -1.494580 & -0.871062 \\
\hline 53 & 6 & 0 & -1.671181 & -2.420136 & -1.889357 \\
\hline 54 & 8 & 0 & -2.654561 & -3.207594 & -2.234845 \\
\hline 55 & 14 & 0 & -4.353516 & -3.273259 & -1.997354 \\
\hline 56 & 6 & 0 & -4.659830 & -4.244940 & -0.432371 \\
\hline 57 & 6 & 0 & -4.924060 & -4.215103 & -3.500451 \\
\hline 58 & 6 & 0 & -5.066193 & -1.552007 & -1.944903 \\
\hline 59 & 8 & 0 & -0.545524 & -2.623494 & -2.526337 \\
\hline 60 & 6 & 0 & -0.419416 & -3.715125 & -3.428028 \\
\hline 61 & 1 & 0 & -0.848368 & -0.812375 & -0.829810 \\
\hline 62 & 1 & 0 & -2.632877 & -1.130432 & -0.523515 \\
\hline 63 & 1 & 0 & -5.726329 & -4.428440 & -0.311007 \\
\hline 64 & 1 & 0 & -4.324021 & -3.723580 & 0.460896 \\
\hline 65 & 1 & 0 & -4.163876 & -5.212832 & -0.465949 \\
\hline 66 & 1 & 0 & -5.996130 & -4.398846 & -3.458598 \\
\hline 67 & 1 & 0 & -4.426030 & -5.179116 & -3.576537 \\
\hline 68 & 1 & 0 & -4.720589 & -3.660693 & -4.414022 \\
\hline 69 & 1 & 0 & -4.943633 & -1.048139 & -0.990177 \\
\hline 70 & 1 & 0 & -6.137485 & -1.605184 & -2.134849 \\
\hline 71 & 1 & 0 & -4.635054 & -0.921033 & -2.720062 \\
\hline 72 & 1 & 0 & -1.140572 & -3.634905 & -4.232990 \\
\hline 73 & 1 & 0 & -0.547863 & -4.654721 & -2.903976 \\
\hline 74 & 1 & 0 & 0.586397 & -3.641121 & -3.816102 \\
\hline 75 & 6 & 0 & 4.824337 & 0.371971 & -0.280216 \\
\hline 76 & 6 & 0 & 5.232042 & -0.835136 & -0.853226 \\
\hline 77 & 6 & 0 & 5.670857 & -1.887137 & -0.074521 \\
\hline 78 & 6 & 0 & 5.697138 & -1.729203 & 1.299652 \\
\hline 79 & 6 & 0 & 5.302074 & -0.548300 & 1.902749 \\
\hline 80 & 6 & 0 & 4.866128 & 0.495893 & 1.110192 \\
\hline 81 & 1 & 0 & 5.186999 & -0.955501 & -1.922306 \\
\hline 82 & 1 & 0 & 5.987177 & -2.817059 & -0.510376 \\
\hline 83 & 7 & 0 & 6.145975 & -2.836152 & 2.125954 \\
\hline 84 & 1 & 0 & 5.337886 & -0.458958 & 2.972875 \\
\hline 85 & 1 & 0 & 4.551557 & 1.413123 & 1.572111 \\
\hline 86 & 6 & 0 & -2.536451 & 4.288354 & 0.771422 \\
\hline 87 & 6 & 0 & -3.723943 & 3.722344 & 1.194655 \\
\hline 88 & 6 & 0 & -4.232001 & 2.643646 & 0.493719 \\
\hline 89 & 6 & 0 & -3.589144 & 2.131175 & -0.620539 \\
\hline 90 & 6 & 0 & -2.402621 & 2.703087 & -1.026995 \\
\hline 91 & 1 & 0 & -2.111229 & 5.107427 & 1.325935 \\
\hline 92 & 1 & 0 & -4.243752 & 4.088445 & 2.060447 \\
\hline 93 & 7 & 0 & -5.456390 & 2.017190 & 0.948926 \\
\hline 94 & 1 & 0 & -4.019564 & 1.302777 & -1.151094 \\
\hline 95 & 1 & 0 & -1.886110 & 2.305951 & -1.882230 \\
\hline 96 & 8 & 0 & 6.226012 & -2.654911 & 3.319556 \\
\hline 97 & 8 & 0 & 6.413683 & -3.880495 & 1.570653 \\
\hline 98 & 8 & 0 & -5.847696 & 1.032466 & 0.354384 \\
\hline 99 & 8 & 0 & -6.023800 & 2.499810 & 1.902824 \\
\hline
\end{tabular}

\section{References}

(1) Wipf, P.; Jung, J.-K. J. Org. Chem. 2000, 65, 6319-6337. 
(2) Jacques, J.; Fourquey, C. Org. Synth. 1989, 67, 1-10.

(3) Simonsen, K. B.; Gothelf, K. V.; Jørgensen, K. A. J. Org. Chem. 1998, 63, 7536-7538.

(4) Tsang, W. C. P.; Schrock, R. R.; Hoveyda, A. H. Organometallics 2001, 20, 5658-5669.

(5) Wu, T. R.; Shen, L.; Chong, J. M. Org. Lett. 2004, 6, 2701-2704.

(6) Zhu, S. S.; Cefalo, D. R.; La, D. S.; Jamieson, J. Y.; Davis, W. M.; Hoveyda, A. H.; Schrock, R. R. J. Am. Chem. Soc. 1999, 121, 8251-8259.

(7) Ishitani, H.; Ueno, M.; Kobayashi, S. J. Am. Chem. Soc, 2000, 122, 8180-8186.

(8) Xue, S.; Yu, S.; Deng, Y.; Wulff, W. D. Angew. Chem., Int. Ed. 2001, 41, 2271-2274.

(9) Gaussian 98, Revision A.11.4.; Frisch, M. J.; Trucks, G. W.; Schlegel, H. B.; Scuseria, G. E.; Robb, M. A.; Cheeseman, J. R.; Zakrzewski, V. G.; Montgomery, Jr. J. A.; Stratmann, R. E.; Burant, J. C.; Dapprich, S.; Millam, J. M.; Daniels, A. D.; Kudin, K. N.; Strain, M. C.; Farkas, O.; Tomasi, J.; Barone, V.; Cossi, M.; Cammi, R.; Mennucci, B.; Pomelli, C.; Adamo, C.; Clifford, S.; Ochterski, J.; Petersson, G. A.; Ayala, P. Y.; Cui, Q.; Morokuma, K.; Salvador, P.; Dannenberg, J. J.; Malick, D. K.; Rabuck, A. D.; Raghavachari, K.; Foresman, J. B.; Cioslowski, J.; Ortiz, J. V.; Baboul, A. G.; Stefanov, B. B.; Liu, G.; Liashenko, A.; Piskorz, P.; Komaromi, I.; Gomperts, R.; Martin, R. L.; Fox, D. J.; Keith, T.; Al-Laham, M. A.; Peng, C. Y.; Nanayakkara, A.; Challacombe, M.; Gill, P. M. W.; Johnson, B.; Chen, W.; Wong, M. W.; Andres, J. L.; Gonzalez, C.; Head-Gordon, M.; Replogle, E. S.; Pople, J. A. Gaussian, Inc.: Pittsburgh PA, 2002. 